\title{
Global analysis of $\mathbf{X}$-chromosome dosage compensation
} Vaijayanti Gupta*, Michael Parisi*, David Sturgill*, Rachel Nuttall ${ }^{\dagger}$, Michael Doctolero ${ }^{\dagger}$, Olga K Dudko ${ }^{\ddagger}$ James D Malley ${ }^{\ddagger}$ P Scott Eastman ${ }^{\dagger \S}$ and Brian Oliver*

Addresses: * Laboratory of Cellular and Developmental Biology, National Institute of Diabetes and Digestive and Kidney Diseases, National Institutes of Health, 50 South Drive, Bethesda, MD 20892, USA. ${ }^{\dagger}$ Incyte Genomics, Palo Alto, CA 94304, USA. ${ }^{\ddagger}$ Mathematical and Statistical Computing Laboratory, Division of Computational Bioscience, Center for Information Technology, National Institutes of Health, Bethesda, MD 20982, USA. ${ }^{\S}$ Current address: Quantum Dot Corporation, Hayward, CA 94545, USA.

Correspondence: Vaijayanti Gupta. Email: vaijayanti@strandls.com; Brian Oliver. Email: oliver@helix.nih.gov

Published: 16 February 2006

Journal of Biology 2006, 5:3 (doi:10.1 186/jbiol30)

The electronic version of this article is the complete one and can be found online at http://jbiol.com/content/5/I/3

(C) 2006 Gupta et al.; licensee BioMed Central Ltd.

This is an open access article distributed under the terms of the Creative Commons Attribution License (http://creativecommons.org/licenses/by/2.0), which permits unrestricted use, distribution, and reproduction in any medium, provided the original work is properly cited.
Received: 20 June 2005

Revised: 30 November 2005

Accepted: 7 December 2005

\section{Abstract}

Background: Drosophila melanogaster females have two $X$ chromosomes and two autosome sets ( $X X ; A A)$, while males have a single $X$ chromosome and two autosome sets ( $X ; A A)$. Drosophila male somatic cells compensate for a single copy of the $X$ chromosome by deploying male-specific-lethal (MSL) complexes that increase transcription from the $X$ chromosome. Male germ cells lack MSL complexes, indicating that either germline $\mathrm{X}$-chromosome dosage compensation is MSL-independent, or that germ cells do not carry out dosage compensation.

Results: To investigate whether dosage compensation occurs in germ cells, we directly assayed $\mathrm{X}$-chromosome transcripts using DNA microarrays and show equivalent expression in $\mathrm{XX} ; \mathrm{AA}$ and $\mathrm{X} ; \mathrm{AA}$ germline tissues. In $\mathrm{X} ; \mathrm{AA}$ germ cells, expression from the single $\mathrm{X}$ chromosome is about twice that of a single autosome. This mechanism ensures balanced $X$-chromosome expression between the sexes and, more importantly, it ensures balanced expression between the single $X$ chromosome and the autosome set. Oddly, the inactivation of an $\mathrm{X}$ chromosome in mammalian females reduces the effective $\mathrm{X}$-chromosome dose and means that females face the same $X$-chromosome transcript deficiency as males. Contrary to most current dosage-compensation models, we also show increased X-chromosome expression in $\mathrm{X} ; \mathrm{AA}$ and $\mathrm{XX}$;AA somatic cells of Caenorhabditis elegans and mice.

Conclusions: Drosophila germ cells compensate for X-chromosome dose. This occurs by equilibrating $X$-chromosome and autosome expression in X;AA cells. Increased expression of the $\mathrm{X}$ chromosome in $\mathrm{X} ; \mathrm{AA}$ individuals appears to be phylogenetically conserved. 


\section{Background}

In most organisms, copy number at any given locus has little effect on proper organismal function. Very few genes are deleterious if present in only one copy (haploinsufficiency) or are overtly deleterious in three copies. Having more or fewer copies (aneuploidy) of a large fraction of the genome is, however, invariably incompatible with viability. For example, over $10 \%$ of human oocytes are aneuploid, but with a few exceptions none of these aneuploid oocytes gives rise to viable offspring [1]. The most common aneuploid genotypes in a wide range of species involve the deletion or duplication of a chromosome or chromosome segment. Deletions are the most deleterious.

In Drosophila melanogaster, a systematic study of aneuploids with deletions of different segments of chromosomes indicates that having only a single copy of $1 \%$ of the genome reduces viability (and often fertility) and having only a single copy of $3 \%$ or more of the genome is lethal [2]. From current estimates of gene content in Drosophila, 3\% represents about 500 genes [3]. Therefore, having only a single copy of 500 genes or more usually results in the collapse of a major part of the genetic network. That genetic networks do indeed collapse because of minor differences in the expression levels of a few connected nodes is evident from genetic interaction studies. In the female germline, for example, the dose of the gene ovarian tumor (otu) strongly modifies the sterility phenotype of flies heterozygous for ovo $o^{D}[4]$. (The gene ovo encodes a transcription factor that acts on otu [5]). Similarly, in the male germline, heterozygosity for haywire or $\beta$-tubulin mutations are tolerated, but heterozygosity for both results in failed spermatogenesis [6].

The sex chromosomes represent an extraordinary exception to the genetic imbalance rule. Drosophila males have one copy of the $\mathrm{X}$ chromosome per diploid set of autosomes (X;AA) and females have two (XX;AA) [7]. As the Drosophila $\mathrm{X}$ chromosome bears about $20 \%$ of the genome [3], Drosophila males vastly exceed the usual 3\% single-copy threshold for viability. This is not due to an underrepresentation of dosage-sensitive genes on the $\mathrm{X}$ chromosome, as females are sensitive to X-chromosome deletions [2]. Therefore, males have a special mechanism(s) to compensate for $\mathrm{X}$-chromosome dose (for reviews see $[8,9]$ ). An extensive set of autoradiographic experiments on the giant polytene chromosomes of the salivary gland showed that the X chromosome in X;AA flies is expressed at roughly twice the level as an $\mathrm{X}$ chromosome in $\mathrm{XX}$;AA flies. Hypertranscription of the $\mathrm{X}$ chromosome in karyotypic males is dependent on a complex of at least five proteins and two non-coding RNAs. The genes encoding the proteins in the complex are referred to as the male specific lethal $(\mathrm{msl})$ loci. Males lacking any of the $m s l$ activities show reduced X-chromosome transcription and die as larvae. At the molecular level, these genes encode a histone-modifying MSL complex, which acetylates histone 4 on lysine 16 (H4 K16). The modification is thought to relieve the general repressive action of histones and result in increased transcription.

Interestingly, the MSLs do not function in the germline. The X chromosomes of male germ cells are not decorated with MSL complexes and are not hyperacetylated at H4 K16 [10]. Furthermore, neither the genes encoding the MSL complex nor the obligate somatic regulators of the MSLs are required for germline viability $[11,12]$. There is similar lack of evidence of dosage compensation in the germline of other organisms $[13,14]$, leading to the hypothesis that germ cells are dosagetolerant. Alternatively, dosage compensation in germ cells may be MSL-independent. Whether the germline X chromosome of Drosophila, or indeed of any organism, is dosage compensated is one of the major unresolved issues in the study of sex chromosomes. Our array results indicate that Drosophila germ cells do, in fact, dosage compensate.

Equally enigmatic are the dosage-compensation systems in Caenorhabditis elegans and mammals, which are based on reducing X-chromosome expression in XX;AA cells $[15,16]$. This is seen most clearly in mammals, where one of the $\mathrm{X}$ chromosomes in XX;AA females is inactivated. In C. elegans, both the $\mathrm{X}$ chromosomes in XX;AA hermaphrodites show reduced expression. In both cases, the dosage-compensation model equilibrates X-chromosome expression between the sexes but it also makes both sexes functionally aneuploid with respect to the autosomes. Both males and females (or hermaphrodites) become functionally X;AA. It has been suggested that this is counterintuitive, as within each diploid $\mathrm{X}$;AA organism, gene expression from a single $\mathrm{X}$ chromosome should be equilibrated and balanced to the autosomes [17-20]. Therefore, it is more useful to think of X-chromosome dosage as a mechanism for equilibrating $\mathrm{X}$ chromosome and autosome expression, rather than as only a mechanism for equilibrating expression between the sexes. This predicts, for example, that in mammals both the single $\mathrm{X}$ chromosome of males and the single active $\mathrm{X}$ chromosome in females are hypertranscribed $[17,18]$. While there is an overwhelming literature supporting X-chromosome inactivation, there has been very little experimental evidence to support hypertranscription of the active $\mathrm{X}$ chromosome [21]. Our examination of array results in C. elegans and the mouse suggests that such X-chromosome hypertranscription does occur.

\section{Results \\ Comparing $\mathrm{XX}$;AA and $\mathrm{X}$;AA expression ratios}

Gene expression in XX;AA and X;AA tissues can be simply visualized by plotting the hybridization intensities of the 
two samples in a scatterplot (Figure 1). There is modest sex-biased expression in the soma, but extensive sex-biased expression in the gonads. In the absence of dosage compensation, we would expect to see XX tissue expression at twice the level of $\mathrm{X}$ tissues. There is no evidence of such a distribution of $\mathrm{X}$-chromosome expression ratios in either tissue. The bulk of the $\mathrm{XX}$ versus $\mathrm{X}$ expression data points overlies the bulk of the AA versus AA expression data points in both tissues. This indicates that the single $\mathrm{X}$ chromosomes of $\mathrm{X} ; \mathrm{AA}$ soma and gonads are expressed at the same level as the two X chromosomes in XX;AA soma and gonads. This strongly suggests that somatic dosage compensation is widespread in adult tissues. More importantly, this provides the first strong evidence that X-chromosome dosage compensation occurs in the germline. It is, however, much more difficult to look for subtle effects of gene dose in the germline because of the dramatic and extensive sex-biased expression observed between ovaries and testes: this sex bias affects about $31 \%$ of the genome and results in a greater than tenfold difference in expression level for some genes.
To avoid distortion due to sex-biased expression, we included sex-transformed tissues in our experiments (see Materials and methods). These tissues were produced by genetic manipulation of the sex-determination hierarchy (Figure 2, and see $[7,22]$ for reviews). We use gonad samples to assay germline dosage compensation. Although both wild-type and transformed gonads are composed of germline and somatic tissues, germline cytoplasm accounts for the bulk of the mRNA $[4,11,23]$. We confirmed that the germline contributes the majority of the transcripts in these hybridizations, as we were unable to extract sufficient RNA from similar numbers of dissected empty gonads [24]. More importantly, we directly verified the composition of the sample gonads by looking for clusters of genes with germline-biased expression and for individual genes with known germline-specific or germline-biased expression patterns (Figure 3). These samples allow us to isolate the effect of X-chromosome dose from the confounding effects of sexual dimorphism.

Scatter in the XX;AA versus $\mathrm{X}$;AA data due to sex-biased expression can be very effectively reduced by sex transformation

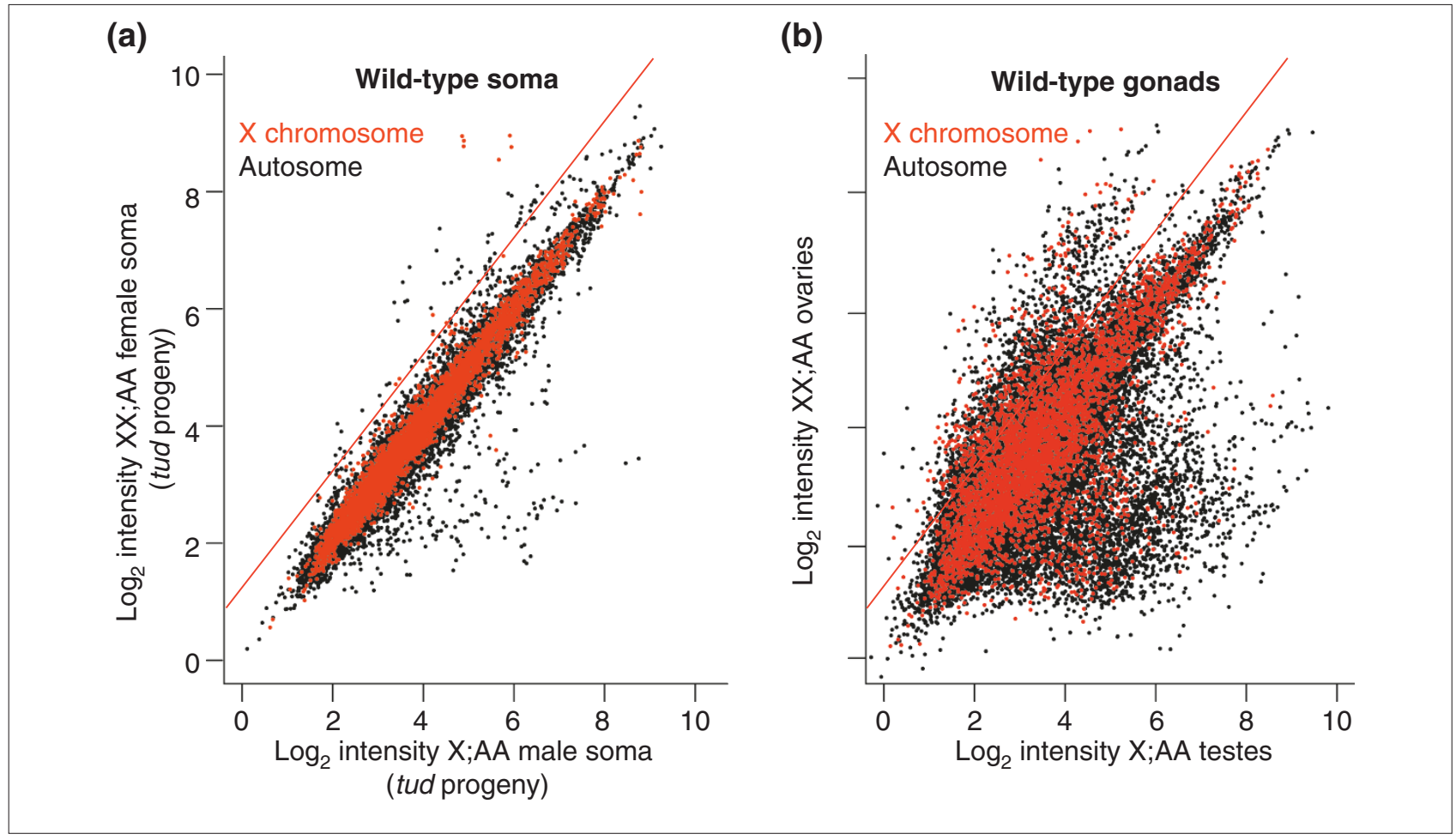

Figure I

Scatterplots of hybridization intensities from wild-type female and male tissues. Hybridization intensities for XX;AA vs $X ; A A$ are plotted along the $y$-and $x$-axis respectively. Data points correspond to elements reporting autosomal genes (black) and X-chromosome genes (red). (a) Germlineless XX;AA female progeny of homozygous tudorl (tud')) mothers (tud $d^{+}$being required for germ cell formation) compared with their germlineless X;AA male siblings; (b) XX;AA wild-type ovaries compared with X;AA wild-type testes from siblings. The expected twofold difference in gene expression in the absence of $\mathrm{X}$-chromosome dosage compensation is shown as a red line. 


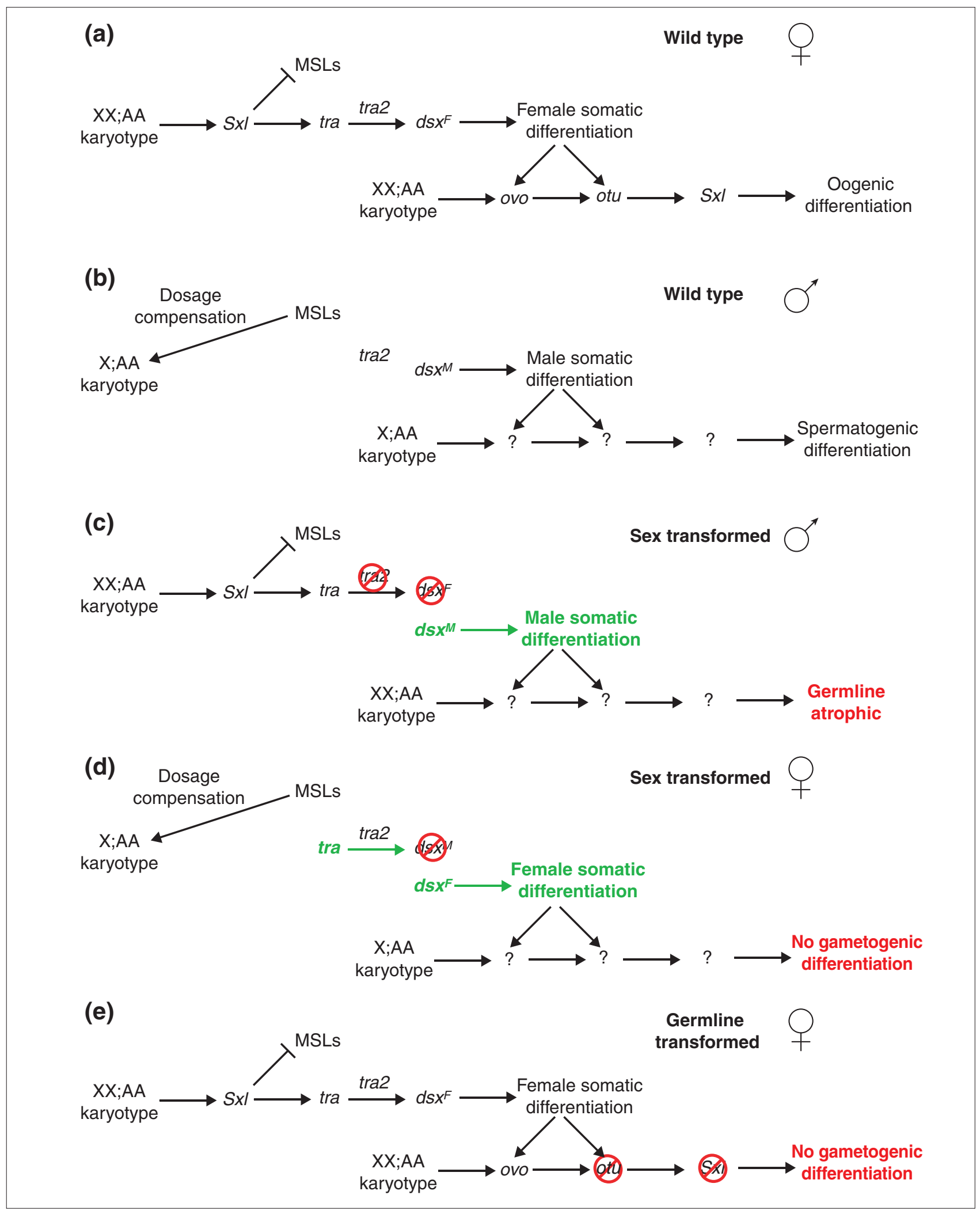

Figure 2 (see legend on the following page) 
(Figure 4). When we compare expression of $\mathrm{XX} ; \mathrm{AA}$ and $\mathrm{X}$;AA soma that were sexually matched (for example, females versus males transformed into females, and males versus females transformed into males), we observe very little high-magnitude differential expression. Even more striking is the similarity in the expression profiles of transformed ovaries bearing non-differentiating germ cells. The high-magnitude differences in expression observed between wild-type $\mathrm{XX} ; \mathrm{AA}$ ovaries and $\mathrm{X} ; \mathrm{AA}$ testes (Figure 1) are nearly abolished when we compared XX;AA and X;AA transformed ovaries (Figure 4). The implications from visual examination of the scatterplots are clear, as hybridization to array elements corresponding to X-chromosome and autosomal genes show expression ratios centered tightly on unity. We confirmed the veracity of this exploratory analysis using multiple statistical tools (see the section Transcriptional response to altered autosomal gene dose, below). This strongly supports the idea that X-chromosome dosage compensation occurs in the germline.

It is formally possible that $\mathrm{X}$ chromosomes are dosage compensated in XX;AA versus X;AA transformed germ cells but not in wild-type oogenic and spermatogenic germ cells. Although most of the data points lie along the diagonal in scatterplots of hybridization intensities comparing XX;AA ovaries and $\mathrm{X} ; \mathrm{AA}$ testes, regression lines for $\mathrm{X}$ chromosome and autosome expression profiles are differentially deflected (Figure 5). This is caused by the large number of genes showing male-biased expression on the autosomes and the lower density of genes with male-biased expression on the $\mathrm{X}$ chromosome [25]. There are, however, many genes with 'housekeeping' functions on both the $\mathrm{X}$ chromosome and the autosomes that are expected to be equally expressed in both oogenic and spermatogenic germ cells.

To determine how genes with housekeeping function respond to dose in the germline, we examined in more detail the expression levels of nuclear genes encoding cytoplasmic or mitochondrial ribosomal subunits [26]. The X chromosome and autosomal genes show strikingly similar distributions ( $p>0.1$ by regression analysis of slopes and intercepts), despite a twofold difference in X-chromosome dose (Figure 5b). We also identified a de facto set of housekeeping genes by investigating array elements showing reduced hybridization variance and high intensity hybridization across all our experiments (Figure $5 \mathrm{c}$ ). In the absence of dosage compensation, we would expect a deficit of X chromosome genes in this subset, as they would show increased variance due to the dose difference. We found no deficit of X-chromosome encoded genes showing lowvariance expression. Both these analyses indicate that at least some X-chromosome genes are dosage compensated in the wild-type germline.

\section{Transcriptional response to altered autosomal gene dose}

To understand the precision of X-chromosome dosage compensation, we first need to understand the general response

Figure 2 (see figure on the previous page)

Sex-determination hierarchy. Sex-biased expression was controlled for by using mutations in sex-determination genes. Relevant aspects of (a) wild-type female, (b) wild-type males, (c) somatic transformation from female to male (sex transformed), (d) somatic transformation from male to female (sex transformed), and (e) germline transformation are outlined. (a) Sex determination occurs in early embryogenesis, before the activation of dosage compensation, which leads to higher levels of expression of transcription factors on the $X$ chromosome in $X X$; $A A$ than $\mathrm{X}$;AA individuals. These transcription factors activate Sex-lethal (SxI) in the soma. The SxI protein regulates the alternative splicing of the transformer (tra) pre-mRNA such that Tra protein is produced only in females. Sxl also inhibits the formation of the MSL dosage compensation complex. Tra protein and non-sex-specifically expressed Transformer2 (Tra2) protein control the alternative splicing of the doublesex (dsx) premRNA. The $d s x$ mRNAs resulting from Tra- and Tra2-mediated splicing encode a female-specific Dsx ${ }^{F}$ transcription factor. Sex determination in the germline is poorly understood and controversial, but a female somatic environment and an independent reading of the $X X$; $A A$ karyotype in germ cells increases expression of positively acting Ovo transcription factors and their direct target, ovarian tumor (otu). The otu locus is required for Sxl activity in the germline. Note that Sxl does not regulate the MSLs in the germline. The female sex-determination hierarchy results in oogenic differentiation. (b) In X;AA flies Sxl protein is not present. This permits the formation of the MSL dosage-compensation complex. The tra pre-mRNAs are spliced to a non-coding form in the absence of $S x l$, and in the absence of Tra protein the dsx pre-mRNA is spliced into a default form encoding a male-specific Dsx ${ }^{M}$ transcription factor. The germ cells develop into sperm. (c) XX;AA flies are transformed from females into males using null mutations of tra2 and by using a $d s x$ mutation encoding a pre-mRNA that is constitutively spliced into the male-specific form $\left(d s x^{s w e}\right)$. Flies bearing $d s x^{s w e}$ in trans to a deletion produce $D s x^{M}$ protein and no Dsx $x^{F}$ protein. Similarly, flies null for tra2 produce only Dsx ${ }^{M}$. To remove germline expression from the analysis of somatic $X$-chromosome dosage compensation we took advantage of the fact that $X X$; $A A$ flies transformed from females into males usually have no germline. These germline-atrophic (having few to no germ cells) $X X$; $A A$ females transformed into males were compared with X;AA male carcasses (everything but the gonads) or with $X ; A A$ males with a genetically ablated germline due to the absence of maternal $t^{+} d^{+}$. (d) X;AA flies are transformed from males into females by expressing female-specific tra cDNA transgenes. The activation of female rather than male sexual differentiation in the X;AA soma results in vast numbers of non-differentiated germ cells, presumably due to sexual incompatibility between the soma and germline. The sexual identity of these cells is ambiguous. (e) Mutations in Sxl (using allelic combinations effecting only in the germline isoforms of Sxl) or otu also result in vast numbers of non-differentiated germ cells. Positive (arrows) and negative (barred lines) genetic or molecular regulation are indicated. Loss-of-function (red) and gain-of-function (green) mutations and phenotypes are indicated. 


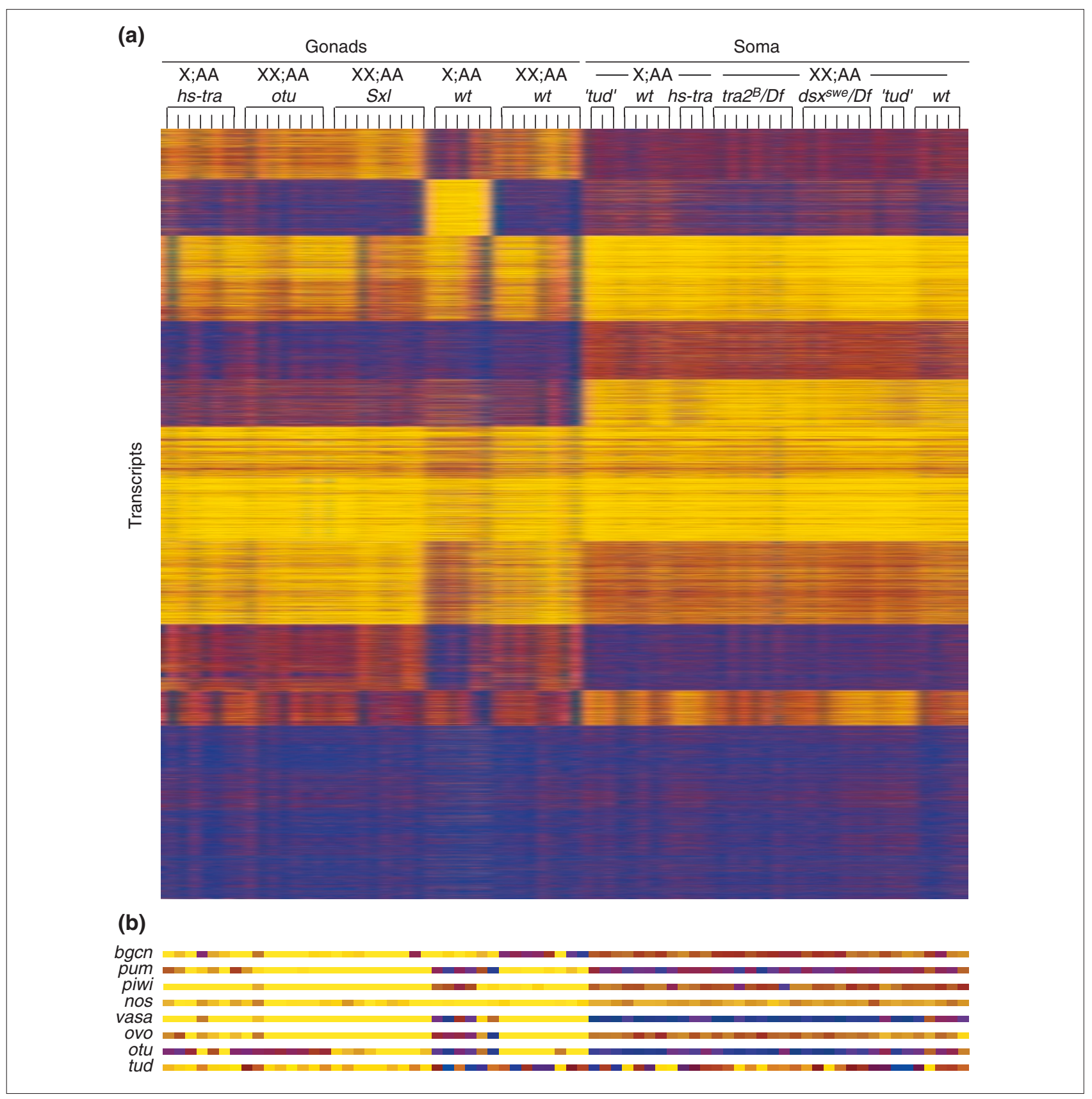

\section{Figure 3}

Germline-biased gene expression in transformed ovaries. (a) Heat diagram (yellow > red > blue) of hybridization intensities for all unique array element sequences $(N=13,267)$ from individual samples (columns) used in this study. Gonad samples (left) and somatic samples (right) are indicated with karyotype (XX;AA and $\mathrm{X} ; \mathrm{AA})$ and abbreviated genotypes; wt, wild type (see Materials and methods for more details). Replicates are indicated (brackets). Germline expression is clearly evident in the gene-expression profiles of transformed ovaries. There are large blocks of elements showing high- or low-intensity hybridization to gonad probes and the opposite pattern when hybridized to samples from carcasses or from flies lacking germ cells but having somatic components of the gonads. (b) Selected genes with known functions in the germline. Array elements representing germlinemarker genes (for example, vasa (vas), pumilio (pum), tudor (tud), piwi and benign gonial cell neoplasia (bgcn) $[67,68]$ ) show strong hybridization to labeled gonad mRNA samples and comparatively weaker hybridization to non-germline samples. Furthermore, at least some of the differences between the samples also support the proposed germline sex-determination pathway. For example, as predicted, both ovo and otu are germlinebiased and overexpressed in XX;AA Sxl ovaries relative to X;AA hs-tra ovaries [40,69]. All these data validate the use of XX;AA and X;AA transformed germlines as matched tissues for the careful analysis of $X$-chromosome dosage compensation in the germline. 


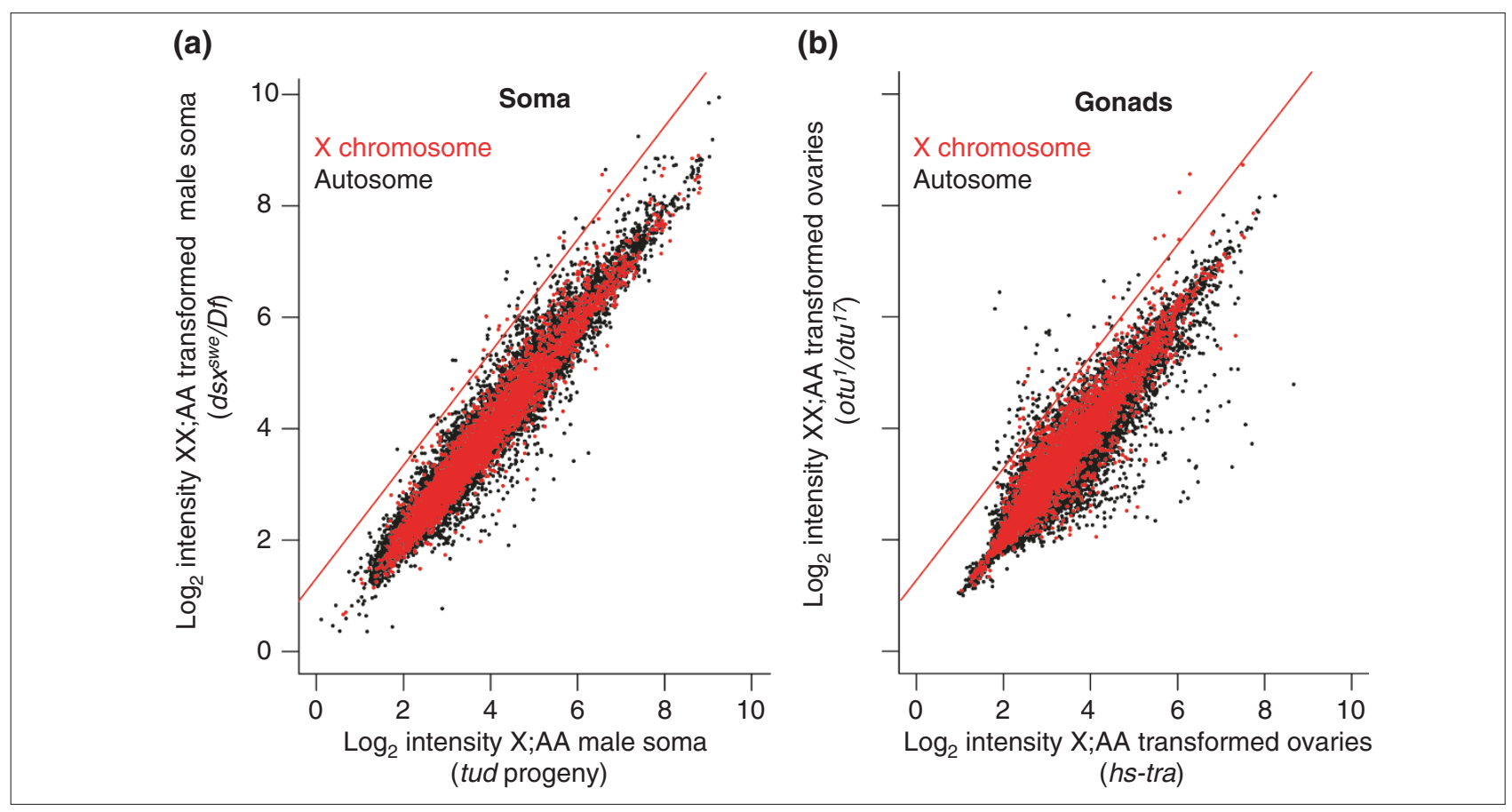

Figure 4

Scatterplots of hybridization intensities from transformed $\mathrm{XX}$;AA and $\mathrm{X} ; \mathrm{AA}$ tissues. Data points correspond to elements reporting autosomal genes

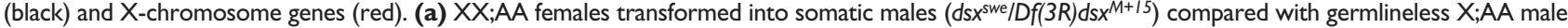
progeny of homozygous tud' mothers and (b) XX;AA ovaries from ovol/otul7 females compared with X;AA ovaries from $h s$-tra ${ }^{83} /+$ males transformed into females. The expected twofold difference in gene expression in the absence of $\mathrm{X}$-chromosome dosage compensation is shown as a red line.

of the biological system to altered gene dose. What should we expect if there is no X-chromosome dosage compensation? A twofold difference in gene dose is unlikely to always result in a twofold difference in steady-state transcript levels, as many genes are regulated by elegant feedback mechanisms that would dampen the effect of gene dose. Similarly, we need to prove that the entire data-handling pipeline allows us to see gene-expression differences associated with altered gene dose.

To determine whether differences in genetic dose result in detectable differences in gene expression, we have directly measured gene-expression changes resulting from altered gene dose due to deficiency (deletion) and duplication of an autosomal segment of the genome. The duplication that we used overlaps the deletion region, but covers more genes. Flies heterozygous for the duplication $D p(2: 2) C a m 3 /+(D p /+)$ have three copies of around 330 genes, while flies heterozygous for the deletion $D f(2 L) J-H /+$ $(D f /+)$ are hemizygous for a subset of around 70 of those duplicated genes [27]. By directly comparing gene expression in flies bearing these aberrations, we assayed the consequences for gene expression of 1-fold, 1.5-fold, and 3-fold differences in gene dose along chromosome arm $2 \mathrm{~L}$.
We isolated mRNA from two independent preparations of females, males and ovaries and performed six hybridizations comparing $D p /+$ and $D f /+$ samples directly against each other on microarrays (Figure 6). The effect of altered autosomal gene dose is obvious in the moving average plots of expression ratios against position along the chromosome arm (Figure 6). We observed distinct alterations of gene-expression ratios between $D p /+$ and $D f /+$ flies within the cytologically defined aneuploid regions in males, females and ovaries. Moving from left to right along such a plot, the ratios are essentially 1.0 (indicating equivalent expression) in the region that is two-copy in both $D p /+$ and $D f /+$ flies, until reaching the region that is three-copy in $D p /+$ flies and two-copy in $D f /+$ flies. At this position there is a strong break in the moving average towards $D p$-biased expression. A similar break in the moving average occurs at the transition to the region that is three-copy in $D p /+$ flies and single-copy in $D f /+$ flies. Altered fold ratios between $D p /+$ flies and $D f /+$ flies return to baseline as the proximal breakpoints of the aberrations are crossed.

The differences in gene-expression ratios corresponding to $1-, 1.5$ - and 3-fold dose changes on chromosome 2L are highly significant $\left(p<10^{-4}\right.$ by ANOVA followed by Tukey 


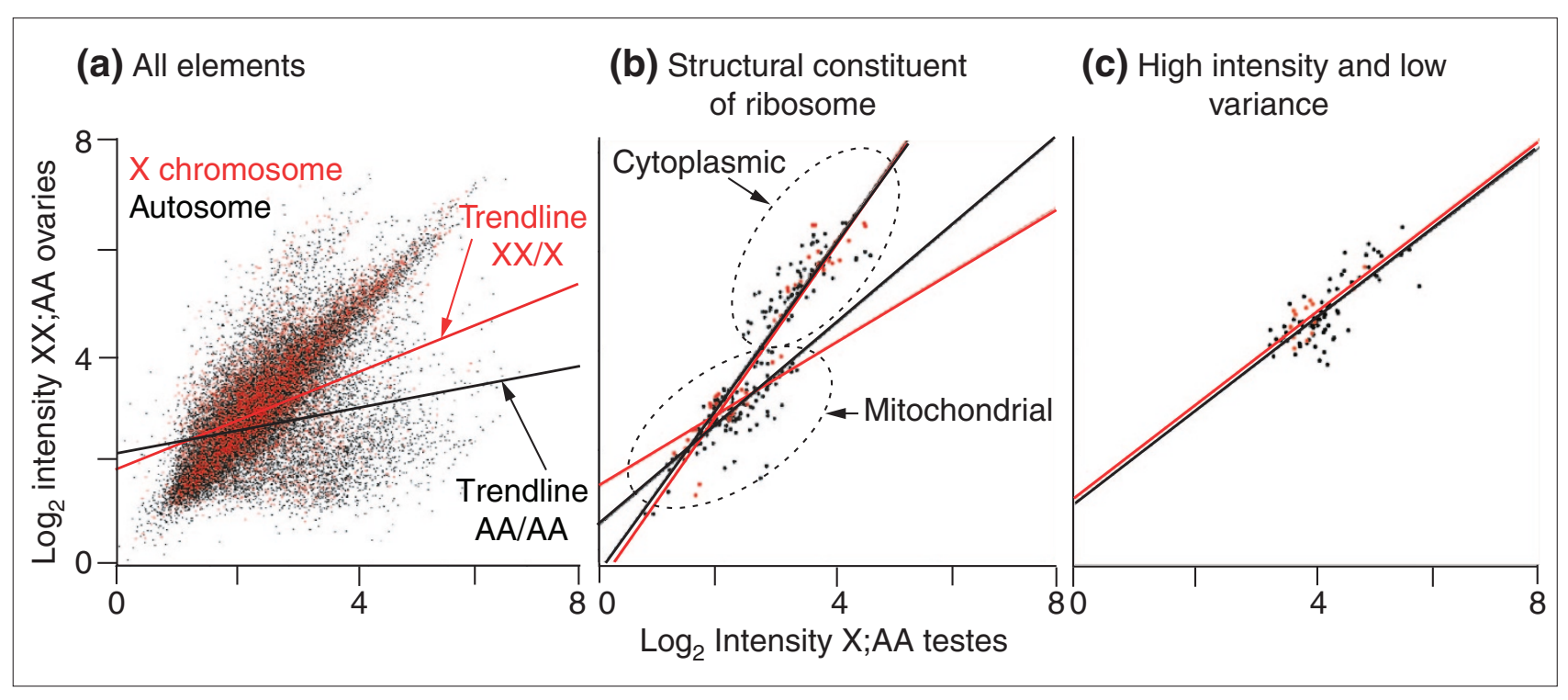

Figure 5

Subsets of gene-expression profiles from wild-type gonads. Scatterplots of hybridization intensities for XX;AA ovary ( $y$-axis ) versus X;AA testis $(x$-axis) samples to elements corresponding to (a) all genes, (b) genes encoding cytoplasmic or mitochondrial proteins, and (c) de facto housekeeping genes (showing high intensity and low variance across all experiments). Data points and regression lines (trendlines) correspond to elements reporting autosomal genes (black) and X-chromosome genes (red).

HSD, Hodges-Lehmann, and Kolmogorov-Smirnov tests; see Materials and methods). No other similar large blocks of genes elsewhere in the genome show significantly greater expression in $D p /+$ flies than in $D f /+$ flies. There were, however, smaller two-copy regions that showed expression ratios mimicking the 1.5 - to 3 -fold gene dose effect or the inverse effect. These regions could be due to unrecognized aberrations or could be secondary transcriptional effects.

We also used these 1-, 1.5- and 3-fold dose-change data to tune our data handling. The on-spot background correction method gave the greatest discrimination power (see Materials and methods and Additional data file 1, available with the online version of this article), and was used for all subsequent distribution analyses. This method maximizes our ability to detect the absence of dosage compensation.

The salient feature of the above analyses is that expression changes due to altered gene dose changes on autosomes are readily detectable. This provides us with a metric for the analysis of the similar X-chromosome gene-expression ratios when comparing XX;AA with X;AA tissues. We performed 48 hybridizations using 96 biological samples (see Materials and methods). Matched XX;AA and X;AA tissues were compared in direct hybridizations and were linked through the loop design to other XX;AA and X;AA samples hybridized on other arrays. This allowed for even greater numbers of comparisons. In all of our global comparisons of XX;AA with X;AA expression in the soma (72 comparisons), a 1.5-fold dose difference on an autosome gave a greater difference in gene-expression profiles than did a twofold difference in X-chromosome dose $\left(p<<10^{-4}\right)$. The same was found in all of our global comparisons of XX;AA to X;AA expression in transformed gonads (119 comparisons). These data conclusively demonstrate that germ cells carry out dosage compensation.

Mechanism of $\mathbf{X}$-chromosome dosage compensation Balancing X-chromosome expression between XX;AA and $\mathrm{X}$;AA germ cells in Drosophila is likely to require either hypertranscription of the $\mathrm{X}$ in males (as occurs in the soma) or hypotranscription of the $\mathrm{X}$ chromosome in females compared with an autosomal reference. Determining which mechanism of dosage compensation operates in the germline requires measuring the absolute expression levels of the $\mathrm{X}$ chromosome genes relative to those of autosomal genes. Gene expression varies by orders of magnitude, but in a large representative population of genes on a chromosome arm we expect that those represented by two copies will have higher transcript abundance than those represented by a single copy.

As a rigorous feasibility test, we examined hybridization intensities in the $D f /+$ region of autosome $2 \mathrm{~L}$. In whole adult males, adult females and ovaries, average hybridization intensities were significantly lower in the 70 or so genes in the single-copy $D f$ segment than in the two-copy 
(a) Analysis of expression ratio:
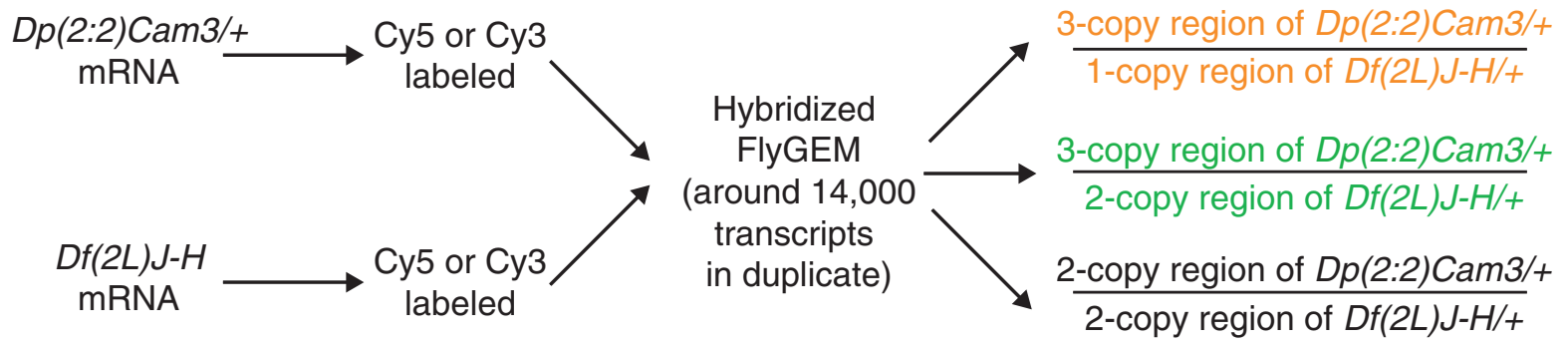

(b)

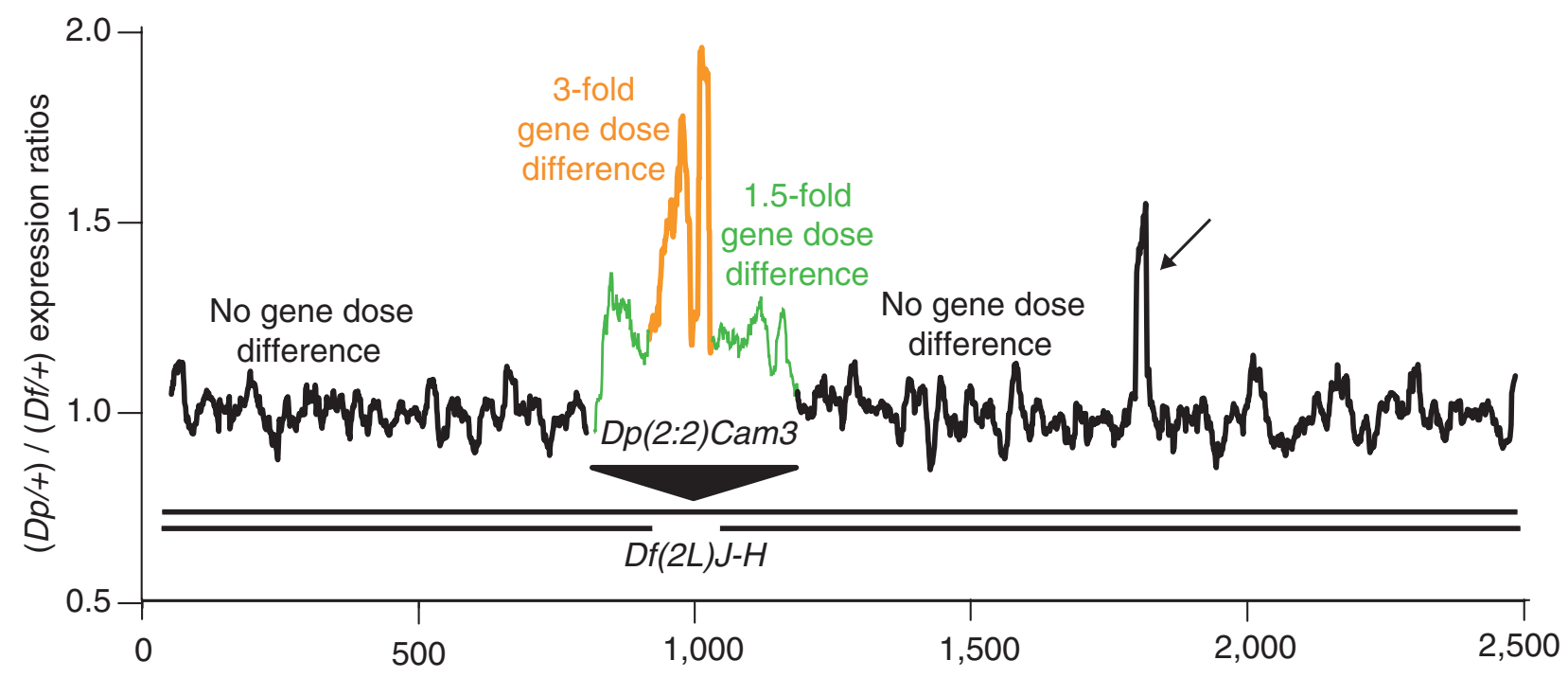

Gene order along chromosome 2L

Figure 6

Gene-expression changes due to an altered gene dose on autosomes. (a) Experimental design to measure gene-expression changes for altered autosomal gene dose. Replicate mRNA samples from flies bearing a duplication, $D p(2 ; 2) C a m 3 /+$, or a deletion, $D f(2 L) J H /+$, on the left arm of autosomal chromosome 2 (2L) were labeled with either Cy3 or Cy5 and hybridized to FlyGEM arrays. These were performed as dye-swap experiments to avoid any effect of dye bias. Following data extraction, changes in expression ratios as a result of differences in the dose of individual genes were determined. There are no differences in underlying gene dose outside the region of the aberrations, and there is a I.5-fold difference when a gene is present on the duplication $(D p)$ or a 3 -fold difference when the gene is present on the duplication and deleted in the deficiency (Df). (b) Average expression ratios from duplicate experiments comparing $D p /+$ vs $D f /+$ adult male flies were plotted as a moving average against gene position along 2L. Gene-expression ratios for no gene dose difference (black), I.5-fold (green) and 3-fold (orange) gene doses are indicated. Breaks in gene-expression ratios occur at the predicted cytological breakpoints of the aberrations. The aberrations are cartooned below the moving average plot, with the $D p$ (triangle) and $D f(g a p)$ indicated. In addition, the diploid regions of the genome where $D p /+$ flies show greater expression than $D f /+$ flies are indicated (arrow).

segments elsewhere in the genome (Figure 7). X chromosomes are hypertranscribed in the soma relative to autosomes. As expected, transcripts from the single $\mathrm{X}$ chromosome in the soma hybridize to arrays as intensely as those from the two-copy autosomes. We observed the same trend in the X;AA germline. Transcripts from the X chromosome of X;AA transformed ovaries show as an high intensity of hybridization as those from the two-copy autosomes. In fact, the $\mathrm{X}$ chromosome appeared to be slightly overexpressed relative to autosomes in the $\mathrm{X} ; \mathrm{AA}$ transformed 


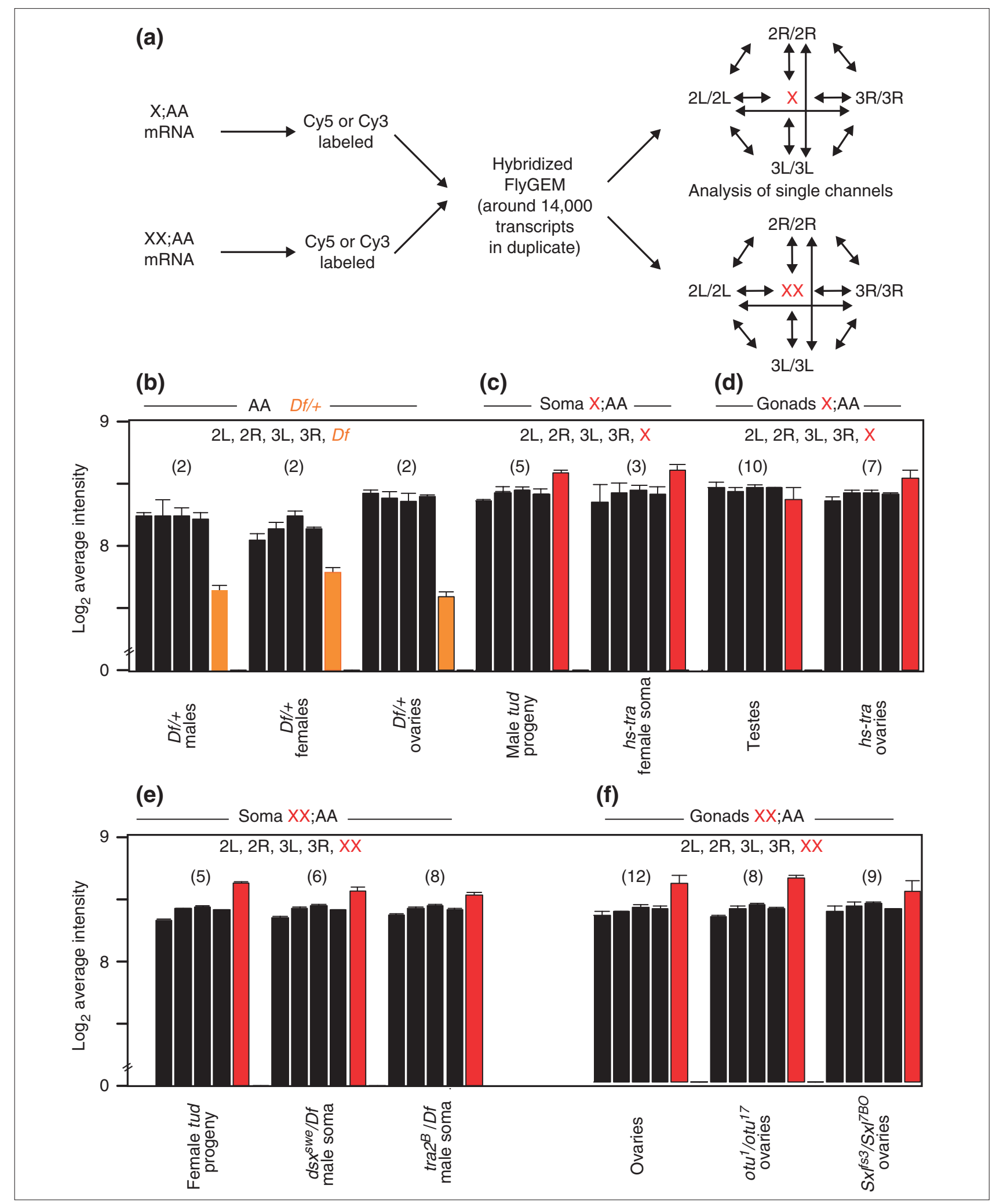

Figure 7 (see legend on the following page) 
ovaries, in each of seven replicate experiments. Even the transcripts from the single $\mathrm{X}$ chromosome in $\mathrm{X}$;AA testis showed higher intensity hybridization than the single-copy genes from any $D f+$ sample $\left(p<<10^{-4}\right)$. These results indicate that $\mathrm{X}$-chromosome dosage compensation in the Drosophila soma and germline is the result of higher percopy X-chromosome transcript accumulation in X;AA cells. Interestingly, the $\mathrm{X}$ chromosome shows slight, but highly significant, overexpression in all XX;AA samples. This may be due to inherent hypertranscription of X-chromosome genes in both males and females.

\section{Escape from dosage compensation}

Although the overall expression of X-chromosome genes appears to be very tightly dosage compensated, there might be small regions or scattered genes that escape dosage compensation. To look for blocks of the $\mathrm{X}$ chromosome that fail to undergo dosage compensation in either the soma or the germline, we plotted a moving average of expression ratios along the length of the $\mathrm{X}$ or the similarly sized autosome arm 2L (Figure 8). In the case of the soma, plots of $(\mathrm{XX} ; \mathrm{AA}) /(\mathrm{X} ; \mathrm{AA})$ expression ratios revealed only noise fluctuating around expression ratios of unity. X-chromosome expression ratios for gonads were slightly skewed towards $\mathrm{XX}$-biased expression, but we detected no overt region of the $\mathrm{X}$ chromosome expressed like a $\mathrm{Df} /+$ region. These results indicate that no contiguous blocks of genes on the $\mathrm{X}$ chromosome escape dosage compensation in the soma or germline.

Scattered genes along the $\mathrm{X}$ chromosome that fail to become dosage compensated in either the soma or the germline are more difficult to detect because there are $\mathrm{X}$ chromosome and autosomal genes that are differentially expressed between $\mathrm{XX}$;AA and X;AA tissues even following sex transformation of those tissues. If some autosomal genes are expressed at higher levels in $\mathrm{XX}$;AA flies than in $\mathrm{X}$;AA flies, then some X-chromosome genes are expected to do the same. To test for isolated genes that have escaped dosage compensation, we extracted all the genes that were at least 1.5-fold overexpressed in either XX;AA or X;AA flies, normalized them to gene content per arm, and looked for departure from a normal distribution (Figure 8). If there are a significant number of genes on the $\mathrm{X}$ chromosome that escape dosage compensation, then there should be more expression outliers mapping to the $\mathrm{X}$ chromosome relative to similarly sized autosomal segments. Furthermore, the outliers from the X chromosome should show XX-biased expression if they escape dosage compensation. In the sextransformed soma, even though there are X-chromosome genes that always show higher expression in $\mathrm{XX}$;AA than $\mathrm{X} ; \mathrm{AA}$ somas, we observed no significant enrichment (Figure 8) of such genes. About twice as many X-chromosome genes as expected, however, show higher expression in XX;AA transformed germline ( $p<10^{-3}$, by the $\chi^{2}$ test). At face value, we can calculate that approximately $2 \%$ of X-chromosome genes escape dosage compensation in the germline. In the soma, sex determination and dosage compensation are linked pathways, however, and this could conceivably be true for the germline $[7,22]$. We cannot rule out the possibility that reduced $\mathrm{X}$-chromosome expression in $\mathrm{X} ; \mathrm{AA}$, hs-tra/+ ovaries relative to XX;AA otu or $S x l$ ovaries is due to a slight defect in germline dosage compensation concurrent with a germline sex transformation.

\section{Dosage-compensation mechanisms in C. elegans and the mouse}

Although X-chromosome dosage compensation equilibrates $\mathrm{X}$-chromosome expression between the sexes, the X;AA individuals are the ones to face an unbalanced karyotype. This makes the increased X-chromosome expression in the $\mathrm{X} ; \mathrm{AA}$ male Drosophila a logical mechanism of dosage compensation. In contrast, X-chromosome transcription is reduced from both $\mathrm{X}$ chromosomes in C. elegans hermaphrodites, and one $\mathrm{X}$ chromosome is inactivated in mammalian females $[15,16]$. In both cases, this dosage compensation equilibrates X-chromosome expression between the sexes, but also makes both sexes functionally aneuploid with respect to the autosomes. This conundrum is solved if $\mathrm{X}$ chromosomes are generally hypertranscribed and if $\mathrm{X}$-chromosome inactivation or hypotranscription in XX;AA females or hermaphrodites is a mechanism to prevent

Figure 7 (see figure on the previous page)

Increased $\mathrm{X}$-chromosome expression in the $\mathrm{X}$;AA soma and germline. (a) Experimental design. The mRNA samples from $\mathrm{XX}$;AA or $\mathrm{X}$;AA somatic or germline tissues were labeled with either $\mathrm{Cy} 3$ or $\mathrm{Cy} 5$ and hybridized to FlyGEM arrays. Hybridization intensities corresponding to $X$ chromosomes (red) and autosomal genes (black) were analyzed within each X:AA or XX:AA sample. (b-f) Histograms of hybridization intensities. (b) Autosomal hybridization intensities from single-copy (orange) and two-copy (black) regions of Dfl+ samples in whole adult males, whole adult females and ovaries. Bars represent autosomal arms, in the order shown across the top. (c-f) Average hybridization intensities from the single $X$ chromosome (red) compared to two copies of each autosomal arms. (c) Germlineless X;AA male progeny of homozygous tud' mothers and carcasses from X;AA males transformed into somatic females with hs-tra. (d) X;AA gonads from wildtype males and X;AA hs-tra sex transformed flies. (e) Germlineless XX;AA female progeny of homozygous tud' mothers and $X X ; A A$ females transformed into males by $d s x^{\text {swe }} / D f(3 R) d s x^{M+15}$ and tra2B/Df(2R)trix. (f) $X X ; A A$ gonads from wild-type and otul7/otul and $S x I^{7 B O} / S x / f 53$ transformed ovaries. Error bars show standard deviation of the mean hybridization intensity from all replicates of each sample. Numbers of hybridization replicates are indicated in parentheses above each histogram panel. 


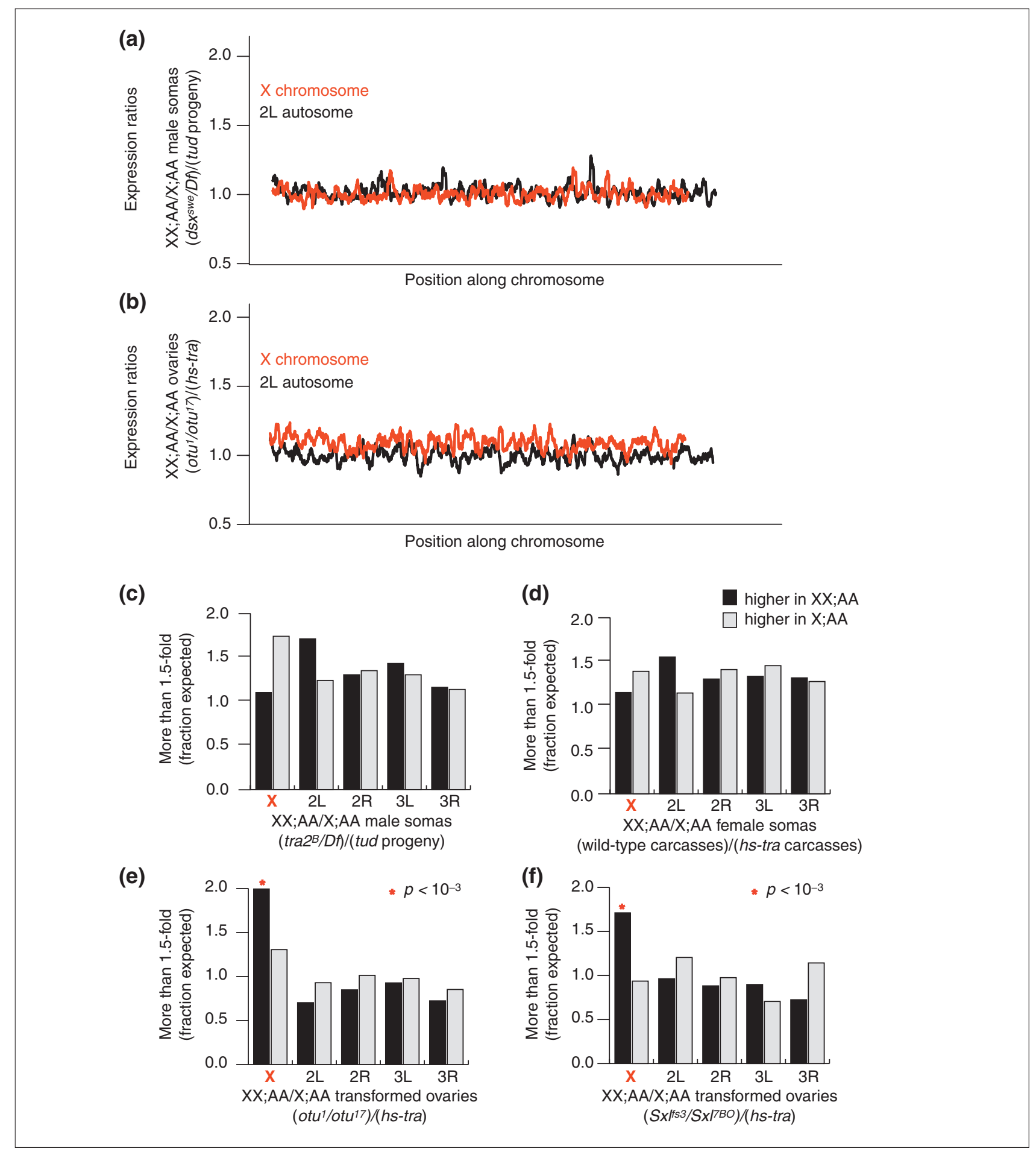

Figure 8

Escaping dosage compensation. (a,b) Moving average of expression ratios plotted against position along the $\mathrm{X}$ chromosome (red) and autosome $2 \mathrm{~L}$ (black) from (a) sex-transformed soma and (b) germline. (c-f) Histograms of genes showing expression ratios greater than I.5-fold in either XX;AA (gray) or X;AA (black) transformed soma (c,d) and germline (e,f). Genotypes are indicated. Genes were analyzed by chromosome arm (number of genes $>1.5$-fold on arm/total on arm)/(number of genes $>1.5$-fold in genome/total in genome). $\chi^{2}$ tests $(p>0.3)$ indicate that there is no enrichment on the $\mathrm{X}$ chromosome for genes expressed more than I.5-fold in XX;AA soma, but that there is enrichment in gonads $\left(*, p<10^{-4}\right)$. 
overexpression of the $\mathrm{X}$ chromosome. Otherwise, hypertranscription of the $\mathrm{X}$ chromosome in $\mathrm{XX}$;AA individuals would result in functional tetraploidy.

In order to investigate the activity level of the $\mathrm{X}$ chromosome in these animals, we reanalyzed previously published array data on gene expression in the soma of hermaphrodite and male C. elegans [28] and female and male mice [29]. These studies reported on gene-expression differences between the sexes rather than dosage compensation. From scatterplots of these data there is little difference between the expression of the $\mathrm{X}$ chromosome in $\mathrm{XX} ; \mathrm{AA}$ versus $\mathrm{X} ; \mathrm{AA}$ germlineless animals or somatic tissues (Figure 9). Given that both species have somatic dosage-compensation mechanisms that equilibrate expression between the sexes, this is unsurprising.

To determine whether per-copy transcription from the $\mathrm{X}$ chromosome is reduced in $\mathrm{XX}$;AA soma, we measured the average hybridization intensity for the $\mathrm{X}$ and all autosomes in both $\mathrm{X} ; \mathrm{AA}$ and XX;AA animals (Figure 9). Transcripts from the single $\mathrm{X}$ chromosome in $\mathrm{X}$ :AA C. elegans male soma hybridize as well as those of two-copy autosomes. Indeed, the $\mathrm{X}$ chromosomes of both $\mathrm{X} ; \mathrm{AA}$ and $\mathrm{XX} ; \mathrm{AA}$ C. elegans appear to be overexpressed relative to autosomes $\left(p<<10^{-4}\right)$. Similarly, X-chromosome transcripts from X;AA and $\mathrm{XX} ; \mathrm{AA}$ mouse soma (average of hypothalamus, kidney and liver) are detected well within the range of those of the autosomes. Many mouse tissues have been examined, and we find evidence of elevated X-chromosome expression in all cases (data not shown). A study concurrent with this one has extensively cataloged mammalian X-chromosome dosage compensation, and confirms our observation that the single $\mathrm{X}$ chromosome in males and the single active $\mathrm{X}$ chromosome in females are compensated [30]. These findings suggest that the single $\mathrm{X}$ chromosomes of $\mathrm{X} ; \mathrm{AA}$ C. elegans and mice are expressed at levels comparable to two autosomes, as we observed in Drosophila. Perhaps this is a universal phenomenon.

\section{Discussion}

Polytene salivary gland chromosomes have greatly facilitated the analysis of dosage compensation in Drosophila [8], but global analysis of dosage compensation in the other tissues has not been conclusive. A recent reverse transcriptase (RT)-PCR study showed a wide range of states of compensation of X-chromosome genes [31]. Sex-biased expression potentially distorts such dosage-compensation analysis, however. There is extensive sex-biased expression in Drosophila [24,32-34] and, furthermore, these genes are not equally distributed between the X chromosome and autosomes [25,35]. Studies directed at subsets of genes have revealed complex relationships between $\mathrm{X}$ chromosome and autosome expression. It is, however, difficult to interpret a subtle difference in $\mathrm{X}$-chromosome expression between $\mathrm{XX}$;AA and $\mathrm{X}$;AA individuals if effects of a similar magnitude are seen for autosomal genes [36]. Global analysis of transcription in non-polytene tissues could help resolve the nature of X-chromosome dosage compensation in the understudied tissues that make up the bulk of the fly.

Microarray analysis is ideally suited to the study of dosage compensation. Our results suggest that remarkably tight $\mathrm{X}$ chromosome dosage compensation occurs in a wide range of adult tissues. Most importantly, we also provide the first evidence for germline dosage compensation in any organism. The compensation mechanism in both the soma and germline is the equilibration of $\mathrm{X}$ chromosome expression with autosomal expression. This is likely to be due to increased expression from the single $\mathrm{X}$ chromosome in $\mathrm{X} ; \mathrm{AA}$, although decreased expression from all the autosomes could also ensure that $\mathrm{X}$ chromosome expression is equilibrated with the autosomes [37].

\section{MSL-independent germline dosage compensation}

Germline X-chromosome dosage compensation has been a black box for decades [22]. As the well-known somatic dosage-compensation mechanisms in the major genetic model organisms clearly do not function in the germline, the idea has gradually emerged that germ cells are dosagetolerant. This is due not to evidence of absence, but to absence of evidence. Although our results show that dosage compensation in the soma and germline are thematically similar, the mechanisms in the germline must be distinct.

The MSL complexes required for dosage compensation in the soma are dispensable in the germline $[10,12]$. This might appear to be non-parsimonious, but germline and somatic gene expression also appear to be distinct. The male and female germline express different types of basal transcription machinery, such as male-germline-specific TATAbox-binding associated factors (TAFs), which might require a different chromatin structure [38,39], and germlinerestricted factors bind the germline-restricted core promoter of the ovo gene [40]. In addition, even though somatic dosage compensation is highly conserved, proteins mediating dosage compensation are not. For example, in some other species of flies the MSL complex associates with all chromosomes, not just the X chromosome [41-43], suggesting that the complex plays no role in dosage compensation in those organisms. In yeast, which lack well differentiated sex chromosomes, one of the main MSL complex components is required for viability [44]. These observations indicate that MSL complexes play markedly different roles in different species. Given the differences in gene expression between the germline and the soma, it is perhaps unsurprising that 


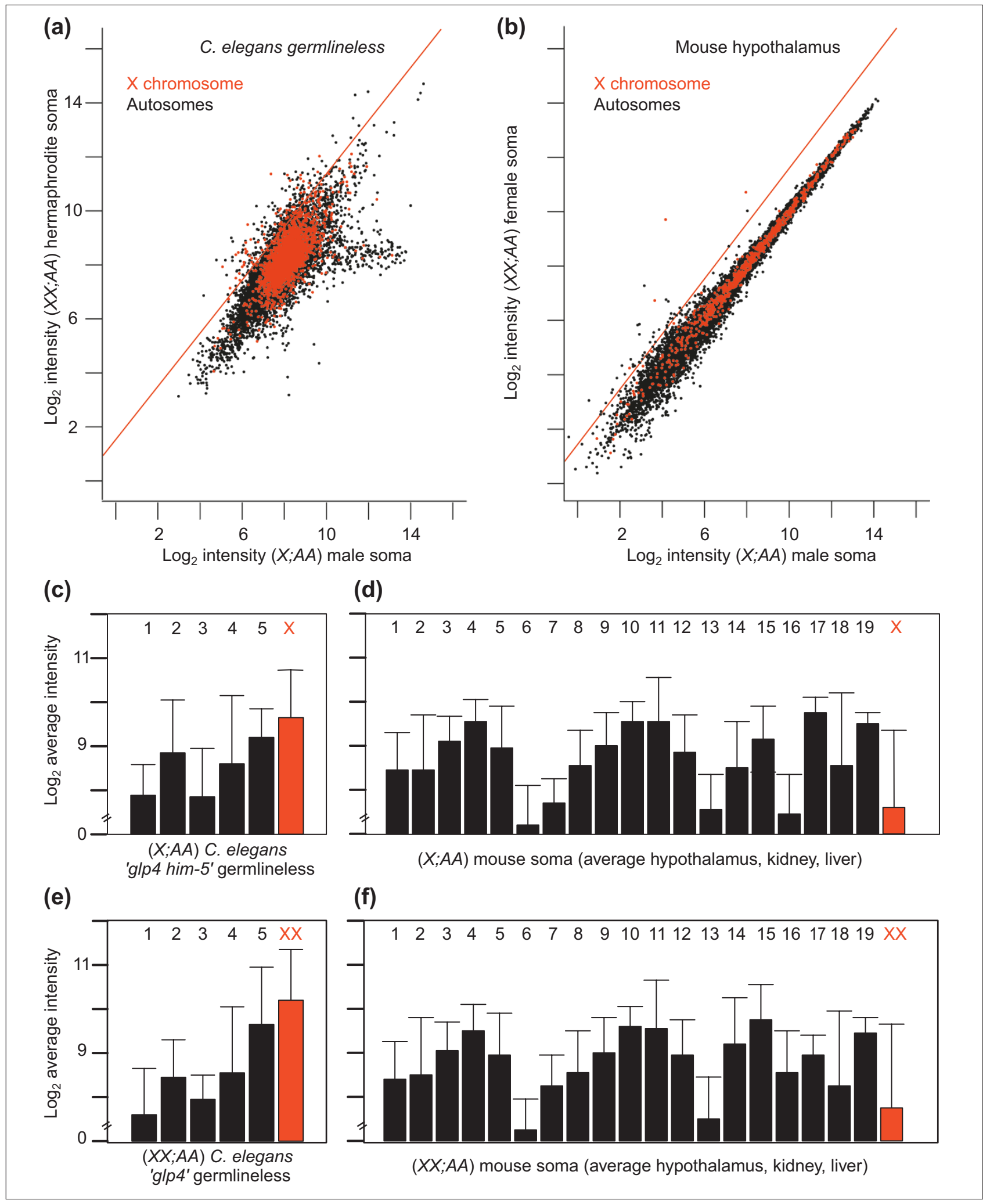

Figure 9 (see legend on the following page) 
different proteins might be involved in Drosophila germline dosage compensation.

It is also unclear whether the MSL complex accounts for all somatic dosage compensation. For example, XX;AA flies lacking Sex-lethal $(\mathrm{Sxl})$ protein in the soma die, presumably because of a failure to repress MSL complex formation [45]. But mutations in the MSL-encoding genes fail to rescue the lethality of XX;AA flies without Sxl, suggesting that Sxl represses additional dosage-compensation functions [46]. Similarly, in polytene salivary glands, large blocks of the X chromosome do not associate with MSL complexes [47]. We find no evidence to support extensive escape from X-chromosome dosage compensation in somatic tissues. These results are consistent with either selective deployment of MSLs at active genes [47] or MSL-independent dosage compensation at a subset of genes.

Mutations in genes regulating germline dosage compensation would be expected to show karyotype-specific phenotypes. Interestingly, there are at least two genes that appear to be required for the viability of $\mathrm{XX}$; $\mathrm{AA}$ but not $\mathrm{X}$;AA germ cells; ovo and stand still (stil) $[48,49]$. Negative and positive Ovo protein isoforms act at the transcription start site of at least some promoters $[5,40,50]$ and Stil protein decorates active chromatin when overexpressed in the soma [49]. Perhaps one or both of these proteins serve to block the upregulation of the $\mathrm{X}$ chromosome that normally occurs in the male germline. This could be analogous to the situation in the Drosophila soma, where dosage compensation is actively repressed in XX;AA females [7]. There are also a number of genes that are required for the viability of male germ cells [51]. Some of these genes may encode dosagecompensation functions. If such dosage-compensation genes exist, they might encode chromatin-modifying complexes, as in the Drosophila soma. Given that our data show that steadystate transcripts from the $\mathrm{X}$ chromosome are compensated, however, there are a host of post-transcriptional mechanisms, such as preferential mRNA stability, that could conceivably mediate germline dosage compensation.

X-chromosome dosage-compensation mechanisms might arise from dosage control mechanisms that are active at many or all genes. Indeed, we have detected transcriptional buffering in our control experiments on the effects of autosome gene dose. We found that the magnitude of the transcriptional effect was less than expected from a simple calculation of gene dose. This inverse effect on transcription relative to gene dose has been known for decades [52-54], and has recently been observed in expression profiling and RT-PCR studies $[31,55-57]$. It seems likely that many genes have a self-contained dosage-compensation system, albeit an imperfect one. The MSL components have general transcriptional activity in yeast and appear to have been co-opted in Drosophila to regulate somatic dosage compensation. Germline dosage compensation might be mediated by other co-opted cis- or trans-acting components that serve to buffer gene expression.

\section{Escape from dosage compensation}

There are $\mathrm{X}$-chromosome genes, such as LSP1- $\alpha$, that escape dosage compensation in Drosophila [58], but it is not known how common this might be. Similarly, in mammals, many X-chromosome genes escape inactivation in females (escape from dosage compensation in $\mathrm{X} ; \mathrm{AA}$ males has not been examined). In theory, this is expected, as not all genes need to be dosage compensated in the course of X-chromosome evolution [59]. Although we can confidently analyze populations of X-chromosome genes, it is quite difficult to determine whether a given gene escapes dosage compensation. Indeed, in the absence of a detailed, gene-by-gene mechanistic study, it might be impossible. There are many autosomal genes that are more highly expressed in XX;AA than X;AA tissues, even after controlling for sex-biased expression. These gene-expression differences are clearly not directly related to X-chromosome dosage compensation. Many of the X-chromosome genes expressed at higher levels in $\mathrm{XX}$;AA than $\mathrm{X}$;AA tissues will be similarly unrelated to dosage compensation per se. In studies of escape from dosage compensation in Drosophila, C. elegans, or mammals where sex-bias is uncontrolled, reports of genes escaping dosage compensation could well be spurious.

On a global level, we find no evidence for escape from dosage compensation in the Drosophila soma. In the germline, however, there are clearly more genes with

Figure 9 (see figure on the previous page)

Scatterplots of hybridization intensities from RNA from somatic tissue from XX;AA and X;AA C. elegans and mouse. Hybridization intensities of (a) germlineless ( $g / p 4) X X ; A A$ hermaphrodites plotted against a population greatly enriched for germlineless $X ; A A$ male $C$. elegans ( $g l p 4$ him5), and (b) female mouse hypothalamus tissue plotted against matched tissue from males. $X$ chromosome (red) and autosomal (black) elements as well as the trend line (red) for the twofold expression difference expected in the absence of dosage compensation are shown. (c-f) Average hybridization intensities corresponding to genes on the $X$ chromosome (red) and autosomes (black) in (c) X;AA glp4 him5 C. elegans male soma and (d) X;AA mouse male soma (average of hypothalamus, kidney and liver) samples, (e) XX;AA glp4 C. elegans hermaphrodite soma, and (f) XX;AA mouse female soma samples. The intensities from individual autosomal arms are averaged across all experiments and standard deviations of the means are indicated. 
XX-biased expression than expected. There are several different interpretations of this finding. It is possible that escape from dosage compensation is more common in the germline (about 2\% of genes). Alternatively, there may be slight under-compensation in the germline. The $\mathrm{X}$ chromosomes in both XX;AA and X;AA gonads appear to be overexpressed relative to autosomes, however. It is therefore possible that the slight excess of XX-biased expression is due to failure to fully dampen $\mathrm{X}$-chromosome hypertranscription in XX;AA females.

\section{Towards a unified model of dosage compensation}

Females have two X chromosomes and males have one. It is therefore natural to think of the problem of dosage compensation as a mechanism to equilibrate $\mathrm{X}$ chromosome expression between the sexes. But changing the dose of large segments of the genome is a problem for the individual with the aneuploid condition - the males in the case of the major metazoan model systems. Remarkably, an X chromosome is inactivated in female mammals. Equally remarkable, both $\mathrm{X}$ chromosomes in C. elegans hermaphrodites are downregulated. Why should $\mathrm{X}$ chromosome dosage compensation be achieved in these organisms by unbalancing gene expression in both sexes? Our analyses of pre-existing array data suggest that both the male $\mathrm{X}$ chromosome and the single active female X chromosome are hypertranscribed or accumulated in mammals. In the course of our study, some of the same data has been reanalyzed independently, and the authors of the other study have reached similar conclusions [30]. These data support a more unified theory of dosage compensation [17-20], whereby X-chromosome expression is increased in all X;AA cells (Figure 10). There may be counteracting forces to increase $\mathrm{X}$-chromosome expression in $\mathrm{X}$;AA males and to keep X-chromosome expression under control in XX;AA females. Indeed, our analysis suggests that in Drosophila and C. elegans, the X chromosomes of XX;AA and X;AA tissues are overexpressed relative to autosomes. Such overshooting would be expected to be more detrimental to $\mathrm{XX}$;AA individuals and could have given rise to $\mathrm{X}$-chromosome inactivation in the mammalian lineage. Interestingly, the repressive chromatin-associated protein HP1 is enriched on the Drosophila X chromosome in males [60]. Perhaps this is to modulate overcompensation.

\section{Conclusions}

Dosage compensation has been under study for a nearly a century. Examination of chromatin structure, rather than direct assay of gene transcription, dominates the recent dosage-compensation literature. Microarray analysis allows us direct access to the transcript accumulation of all X-chromosome genes. The Drosophila array data provide the first demonstration in any organism that germ cells dosage compensate.

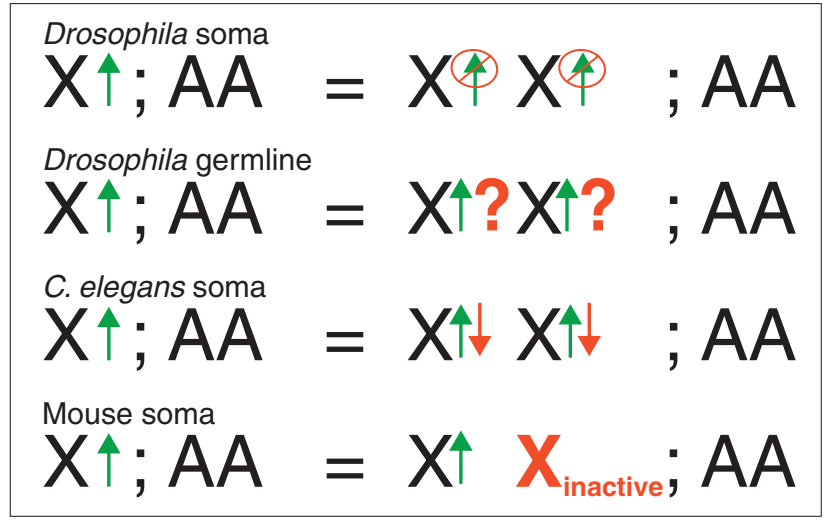

Figure 10

Model for X-chromosome dosage compensation in Drosophila, C. elegans and mouse. $\mathrm{X}$-chromosome transcription is generally upregulated (green arrow). X;AA tissues thus avoid unbalanced expression of $\mathrm{X}$-chromosome genes. In order to avoid overexpression of $\mathrm{X}$-chromosome genes (red symbols), females either destroy the compensation machinery used in males (Drosophila soma), or deploy a counteracting mechanism to reduce expression from both hyperactive $X$ chromosomes ( $C$. elegans) or eliminate expression from one hyperactive $X$ chromosome (mouse). The mechanism used in the XX;AA Drosophila germline is unknown, but given that $\mathrm{X}$-chromosome expression in the female germline is bi-allelic, an X-inactivation mechanism is unlikely.

These data also add a significant twist to our understanding of dosage compensation in mammals and C. elegans. Microarray analysis will be a vital tool for directly assaying this intriguing whole-chromosome regulation of gene expression.

\section{Materials and methods Drosophila strains}

Flies were grown at $25^{\circ} \mathrm{C}$, aged for 5-6 days, dissected, flashfrozen, and prepared for RNA extraction as described previously [24] (see the Flybase compendium of information on genes, alleles, and phenotypes for additional descriptions [27]). Flies used to test for the effect of chromosomal aberrations were $D p(2 ; 2) C a m 3 /+$ and $D f(2 L) J-H /+$ that were generated from outcrossing the stocks to $\gamma^{1} w^{67 c}$ to remove the balancer chromosomes. In addition to using $\mathrm{XX} ; \mathrm{AA}$ females $\left(y^{1} w^{67 c}\right)$, we transformed XX;AA flies into males using null mutations of transformer2 (tra2 $2^{B} / D f(2 R)$ trix) and by using a $d s x$ mutation encoding a pre-mRNA that is constitutively spliced into the male-specific form $\left(d s x^{\text {swe }}\right)$. Flies bearing $d s x^{\text {swe }}$ trans to a deletion produce $\mathrm{Dsx}^{\mathrm{M}}$ protein and no Dsx ${ }^{\mathrm{F}}$ protein $\left(d s x^{\text {swe }} / \mathrm{D} f(3 R) d s x^{\mathrm{M}+15}\right)$. Similarly, flies null for tra2 produce only Dsx ${ }^{\mathrm{M}}$. To obtain X;AA flies that are sex transformed from males into females, we crossed female flies bearing female-specific tra cDNA transgenes $\left(w ; P\left\{w^{+}\right.\right.$ hs-tra $\left.{ }^{83}\right\} / C \gamma O$ or Gla) or (Df(3L)st $t^{7}$ Ki roe $p^{p} P\{h s-t r a\} / T M 3$ or TMG) to $B^{s} Y$ males. The hs-tra transgenes are sufficiently 
active at $25^{\circ} \mathrm{C}$ to transform males into phenotypic females. We did not utilize a heat-shock regimen.

In order to study the expression changes resulting from a twofold change in the dose of the $\mathrm{X}$ chromosome between the XX;AA and X;AA germlines, we utilized genetic mutations that we show remove most sex-biased germline expression from the analysis. The activation of female rather than male sexual differentiation in the soma with hs-tra in $\mathrm{X}$;AA flies results in gonads with vast numbers of poorly differentiated germ cells. Mutations in $S x l\left(y S x l^{f s 3} / y \mathrm{~cm} \mathrm{Sxl}{ }^{7 B O}\right)$ or otu (ctotu $\left.v^{24} / y w o t u^{17}\right)$ result in a similar germline phenotype. Occasionally, a few ovaries from X;AA $h s$-tra flies and germline-transformed $S x l$ or otu flies bear eggs. These ovaries were not included in the samples. Similarly, very small ovaries that are essentially germlineless were not included in any of our samples.

To remove germline expression from the analysis of somatic $\mathrm{X}$-chromosome dosage compensation, we took advantage of the fact that XX;AA flies transformed from females to males usually have no germline, but rarely have a few germ cells showing either oogenic or spermatogenic phenotypes. These germline-atrophic XX;AA females transformed into males were compared with both gonadectomized X;AA males or sham-dissected X;AA males with a genetically ablated germline as a result of the absence of maternal $\mathrm{Tud}^{+}$ (progeny of $t u d^{1} b w^{1} s p^{1}$ mothers).

\section{Arrays}

We have used an extensively tested microarray platform designed to detect transcription from $94 \%$ of D. melanogaster release 1 genes [61]. Unlike most array studies, where the object is to determine which genes are altered between tissues, life stages or treatments, we focus here on non-differential expression. In dozens of homotypic hybridization experiments (where an mRNA sample is split, labeled with either Cy3 or Cy5, mixed, and hybridized to the array) performed as part of this (data not shown) and previous studies, we find that the expression ratio is 1 and that there are very few outliers $[24,25,61]$. In the most extensive set of such homotypic hybridizations, the $99.5 \%$ confidence intervals were always between 1.4 and 1.5-fold [61]. Given that only a handful of genes are expected to show artifactually a greater than 1.5-fold expression difference, we easily had the sensitivity to detect changes in expression owing to a twofold difference in the 2,245 $\mathrm{X}$ chromosome genes represented on the array.

We have further shown that twofold differences in mRNA concentrations can be readily detected by adding known concentrations of mRNA to hybridization mixes in 'spike-in' experiments [61]. Analysis of spike-in control data (a twofold change in input results in a twofold difference in expression ratios) and a comparison of sex-biased expression determined by FlyGEM and northern blotting reveal no evidence of compression of expression ratios in our data [24,61].

\section{Sample preparation and labeling}

We used 96 biological samples in this study. Careful sample preparation ensures minimum adjustment in subsequent data handling. Dissections, flash freezing and RNA extractions were performed as described [24,61]. Briefly, total RNA was extracted using Trizol (Life Technologies, Carlsbad, USA), followed by mRNA isolation using an Oligotex poly(A) extraction kit (Qiagen, Valencia, USA). RNA concentration was determined using RiboGreen dye (Molecular Probes, Oak Ridge, USA) in a fluorescent assay using a Luminescence Spectrometer LS-50B (PerkinElmer, Fremont, USA) fitted with $485 \mathrm{~nm}$ (excitation) and $520 \mathrm{~nm}$ (emission) filters. RNA quality was determined by capillary electrophoresis on a Bioanalyzer 2100 (Agilent, Palo Alto, USA), using the 6000 Nano Assay kit (Agilent) according to the manufacturer's instructions.

Samples were labeled with Cy3- or Cy5-labeled random nonamers (Trilink Biosciences, San Diego, USA). To ensure that each of the nonamer sets was in fact equally 'random', we ordered a single batch of random oligonucleotides that was split into half at the penultimate synthetic step with Cy3 or Cy5 nucleotides added at the end. To verify performance of these oligonucleotides, we performed an experiment in which RNA was labeled using both Cy3 and Cy5 nonamers in the same tube, followed by hybridization, scanning and analysis. There was no dye bias evident. Hybridizations of samples to the microarrays were performed at $60^{\circ} \mathrm{C}$, followed by washes exactly as described [24,61].

\section{Scanning}

Arrays were scanned using an Axon GenePix 3000A fluorescence reader (Molecular Devices Corporation, Union City, USA) in which the photon multiplier tube (PMT) settings were first adjusted using calibration slides. GenePix v.4.1 image acquisition software (Molecular Devices Corporation) was used to extract signal for each target element. Calibration slides were Ultra GAPS (Corning, Acton, USA) spotted with $10^{-3}$ to $10^{-6}$ dilutions of a $0.33 \mathrm{nM}$ each stock of Cy3 and Cy5 dyes (Amersham Biosciences, Pittsburgh, USA) using GMS 417 Arrayer (Affymetrix, Santa Clara, USA). The PMT voltages, in the linear range, were balanced using these slides. With each new batch of labeling and hybridization, we included a homotypic hybridization [61], in which the same RNA preparation from $y^{1} w^{67 c}$ whole males was split into two tubes, labeled with Cy3 or Cy5 nonamers, mixed and hybridized. Slight adjustments of PMT voltages were sometimes made on these control slides. No elements were 
saturated. Once PMT settings were determined, all arrays in the same hybridization batch were scanned at the same voltage settings. Hybridization and labeling quality was determined by confirming that the channels were close to global balance in the absence of PMT adjustment (no 'onthe-fly' adjustments were made). No arrays were discarded because of failed labeling reactions in one of the two channels. In addition, all elements were printed in duplicate. Regression analysis of plots of duplicate elements was also used as a quality-control step for gradients and severe speckling. No arrays were discarded following duplicate element evaluation. Finally, we determined the fraction of elements that returned signal above on-spot background (see Data handling). Three arrays were discarded from the study because less than $50 \%$ of elements exhibited above-background signal.

\section{Loop design for microarray pairings}

Samples from all flies and tissues were prepared (Table 1) and hybridized using a loop design [62]. This design (see Additional data file 2) ensured first, that the highest-quality direct hybridizations were predominantly between matched $\mathrm{XX} ; \mathrm{AA}$ and X;AA tissues and second, that any sample could be compared with every other sample included in the study, after normalization across all samples. Most of the relevant samples were compared directly, but all samples are connected in the design for indirect comparisons. In most direct comparisons we have included both technical labeling replicates (dye-flips) and biological replicates. Although we used a series of pair-wise comparisons of XX;AA and X;AA samples in this study, the flexible design will allow easy future use to address other questions about sex-biased expression.

\section{Data handling}

Drosophila data were processed using the Bioconductor [63] package limma (Linear Models for Microarray Analysis) v. 1.7.6, to adjust for effects that arise from variations in microarray technology rather than biological differences. Because of the care taken in using identical RNA concentrations in labeling reactions and scanner settings, data handling resulted in minimal adjustments to the raw data. We did not average duplicate array elements as this would reduce statistical power in later steps. At the individual array level, the raw intensity data were normalized using print-tip loess (locally weighted scatter plot smooth) [64]. This corrects for tip biases and for minor hybridization gradients. This was followed by one of three different background corrections. For background elimination, intensities less than the average intensities of the barcode elements (designed against DNA corresponding to an intron that were included in printing plates for tracking purposes, but are also quite useful for background correction [61]) were classified as background and excluded from calculations. For on-spot background correction, the average intensity of the barcoding elements in the array were subtracted from the spot intensity for every array element. For the no-correction option, we used quantile normalization to compare across arrays [64]. Experiments with the $D f /+$ and $D p /+$ flies confirm that on-spot background correction maximizes expression differences within aneuploid segments, but the effect of the copy number was evident with both background elimination and no background correction (see Additional data file 1).

Array data from C. elegans and mouse were extracted directly from the Gene Expression Omnibus (GEO) website [65]. We performed no additional normalization or background correction. The C. elegans experiments were two-channel hybridizations on spotted arrays [28]. Test samples were hybridized with a reference sample. The reference channels cancel in our comparison of XX;AA versus X;AA gene expression. The mouse experiments were performed on Affymetrix arrays [29].

\section{Data sources}

All array data are available at GEO. An extensive platform description of FlyGEM is available at GEO under accession number GPL20 [61]. Data are available under GEO accessions GSM37438-GSM37451， GSM2464-2467， GSM16582GSM16583, GSM16570-GSM16581, and GSM77749GSM77752. Expression data from mouse hypothalamus, kidney and liver were obtained from GEO accession number GSE1148 [29]. C. elegans data were from GEO accession number GSE715 and GSE724 [28].

\section{Exploratory data visualization}

Data were clustered and visualized using Cluster 3.0/TreeView 1.6 [66]. A self-organizing map (SOM) was calculated to organize normalized hybridization intensities (on-spot background subtraction followed by quantile normalization across arrays) of array elements at 100,000 iterations, followed by k-means clustering of genes with 10 nodes using the correlation (uncentered) similarity metric. Genes, but not experiments, were clustered. We averaged duplicate elements for this analysis to avoid crashing the application.

For scatterplots and moving average plots, we used data processed by background elimination followed by quantile normalization across all experiments. We used on-spot background corrected data for histograms of Drosophila expression data. Chi-squared tests were performed in Excel (Microsoft, Redmond, USA).

Array elements encoding Drosophila ribosomal subunits were identified using the Gene Ontology identifier 'structural constituent of ribosomes' as assigned in GEO accession GPL20. The de facto housekeeping elements were identified as follows. Following background elimination and quantile 
Table I

\section{Samples used for measuring $\mathbf{X}$ chromosome dosage compensation}

\begin{tabular}{|c|c|c|c|c|c|}
\hline Karyotype & Somatic phenotype & Germline phenotype & Sample & Genotype & $N$ \\
\hline$X X ; A A$ & Female & Oogenic & Wild type (whole fly) & $y^{\prime} w^{67 c} /+; D p(2 ; 2) C a m 3 /+$ & 2 \\
\hline$X X ; A A$ & Female & Oogenic & Wild type (whole fly) & $y^{\prime} w^{67 c} /+; D f(2 L) J-H /+$ & 2 \\
\hline$X X ; A A$ & Female & Oogenic & Wild type (ovary) & $y^{\prime} w^{67 c} /+; D p(2 ; 2) C a m 3 /+$ & 2 \\
\hline $\mathrm{XX}$;AA & Female & Oogenic & Wild type (ovary) & $y^{\prime} w^{67 c} /+; D f(2 L) J-H /+$ & 2 \\
\hline X;AA & Male & Spermatogenic & Wild type (whole fly) & $y^{\prime} w^{67 c} / Y ; D p(2 ; 2) C a m 3 /+$ & 2 \\
\hline X;AA & Male & Spermatogenic & Wild type (whole fly) & $y^{\prime} w^{67 c} / Y ; D f(2 L) J-H /+$ & 2 \\
\hline$X X ; A A$ & Female & Oogenic & Wild type (whole fly) & $y^{\prime} w^{67 c}$ & 5 \\
\hline$X X ; A A$ & Female & Ovaries removed & Wild type (carcass) & $y^{\prime} w^{67 c}$ & 8 \\
\hline$X X ; A A$ & Female & Oogenic & Wild type (ovary) & $y^{\prime} w^{67 c}$ & 12 \\
\hline$X X ; A A$ & Female & Germlineless & tud' progeny (whole fly) & tud $b w^{\prime} s p^{\prime}$ & 5 \\
\hline $\mathrm{XX}$;AA & Transformed to male & Atrophic & $d s x^{\text {swe }} / D f$ (whole fly) & $d s x^{s w e} / D f(3 R) d s x^{M+15}$ & 6 \\
\hline$X X ; A A$ & Transformed to male & Atrophic & $\operatorname{tra} 2^{B} / D f$ (whole fly) & tra $2^{B} / D f(2 R)$ trix & 8 \\
\hline$X X ; A A$ & Female & $\begin{array}{l}\text { Non-differentiated } \\
\text { 'ovarian tumor' }\end{array}$ & otu ${ }^{17 / o t u^{\prime}}$ (ovary) & ct otul $v^{24} / y$ w otu 17 & 8 \\
\hline$X X ; A A$ & Female & $\begin{array}{l}\text { Non-differentiated } \\
\text { 'ovarian tumor' }\end{array}$ & Sx/fs3/Df (ovary) & $y S x f^{f s 3} /\left.y c m S x\right|^{7 B O}$ & 9 \\
\hline X;AA & Male & Spermatogenic & Wild type (whole fly) & $y^{\prime} w^{67 c}$ & 9 \\
\hline X;AA & Male & Testes removed & Wild type (carcass) & $y^{\prime} w^{67 c}$ & 7 \\
\hline X;AA & Male & Germlineless & tud' progeny (whole fly) & tud' $b w^{\prime} s p^{\prime}$ & 5 \\
\hline $\mathrm{X} ; \mathrm{AA}$ & Transformed to female & Ovaries removed & hs-tra (carcass) & $y^{\prime} w^{\left.67 c / Y ; D f(3 L) s t^{+7} \text { Ki roe } p^{p} P\{h s-t r a\} /+\right)}$ & 2 \\
\hline X;AA & Transformed to female & Ovaries removed & hs-tra (carcass) & $w / B^{s} Y ; P\left\{w^{+} h s-t r a^{83}\right\} /+$ & 1 \\
\hline X;AA & Male & Spermatogenic & Wild type (testis) & $y^{\prime} w^{67 c}$ & 10 \\
\hline X;AA & Female & $\begin{array}{l}\text { Non-differentiated } \\
\text { 'ovarian tumor' }\end{array}$ & hs-tra (ovary) & $y^{\prime} w^{\left.67 c / Y ; D f(3 L) s t^{7} \text { Ki roe } p^{p} P\{h s-t r a\} /+\right)}$ & 4 \\
\hline X;AA & Female & $\begin{array}{l}\text { Non-differentiated } \\
\text { 'ovarian tumor' }\end{array}$ & hs-tra (ovary) & $w / B^{s} Y ; P\left\{w^{+} h s-t r a^{83}\right\} /+$ & 3 \\
\hline
\end{tabular}

normalization across all experiments, we selected the elements with hybridization intensities greater than two standard deviations above the median intensity of all array elements, from all experiments. From the above, we selected the de facto housekeeping elements as those elements with standard deviations for the expression ratios as \pm 0.1 . Regressions for the X-encoded and the autosomeencoded elements were calculated separately in Bioconductor. Slopes and intercepts were compared at the 95\% confidence limit.

Moving averages of expression ratios for every 40 consecutive elements (two elements per predicted transcript) were plotted against the corresponding gene positions on chromosome arm $2 \mathrm{~L}$ or X. The predicted cytological break points [27] were used to define the boundaries of aneuploid segments on chromosome arm $2 \mathrm{~L}$, in the $D p /+$ vs $D f /+$ experiments. Moving averages were calculated in Bioconductor.

\section{Analysis of distributions}

The distribution statistics cited in the text were obtained using data processed with print-tip loess and on-spot background correction on individual arrays, and quantile normalization between arrays. We can unequivocally show that a modest 1.5-fold difference in gene dose has a detectable effect on gene expression using precisely the same data-handling methods 
that we apply to the study of the X. In addition, the data-handling method used is the least favorable method vis-a-vis the conclusions we report. Differences in gene expression in autosomal aneuploid segments were always significantly greater than those observed between the $\mathrm{X}$ chromosomes from XX;AA and X;AA samples.

We performed a one-way analyses of variance (ANOVA) followed by the Tukey HSD method for protection in multiple comparisons using Matlab (The MathWorks, Natick, USA). All cells were filled ten times. One-way ANOVA was used to compare three different sets of expression ratios or hybridization intensities. The single blocking factor for one-way ANOVA was gene dose. We used bootstrap sampling of pooled array data to fill each of the three ANOVA cells of the one-way design. For ratiometric data, those cells were $(D p /+) /(+/+), \mathrm{AA} / \mathrm{AA}$, and $\mathrm{XX} / \mathrm{X}$ (see Additional data file 3, Tables 1-3). To fill the $(D p /+) /(+/+)$ cell, we pooled expression ratios associated with a 1.5 -fold difference in gene dose from the replicate samples from whole males, whole females and ovaries, and sampled 600 ratios. There are fewer genes in the $D p$ region than on all autosomes or on the $\mathrm{X}$ chromosome. Therefore, the stability of this inference was verified by filling this cell with 200 ratios from each of the tissue types separately (data not shown). To fill the AA/AA cell we pooled all array data reported in this article where the gene dose was 1 and sampled 600 ratios. To fill the XX/X cells we sampled 600 X-chromosome ratios for each of three different gonad comparisons separately. Those are $\left(\mathrm{otu}^{1} / \mathrm{otu}^{17}\right) /($ hs-tra $/+)$, $\left(S x l^{53} / S x l^{7 B O}\right) /(h s-t r a /+)$ and ovary/testis (see Additional data file 3, Tables 1-3). For analysis of Drosophila intensity data, the cells were $D f /+, A A$, and X (see Additional data file 3, Tables 4,5). As with ratiometric data, intensity data were bootstrap sampled 600 times and the procedure was repeated ten times. Stability for the $D f /+$ cell was also verified. The inferences were similar in all ten samplings of 200 intensity values from each tissue type (data not shown). For C. elegans and mouse data the cells were AA and X (see Additional data file 3, Table 6). These cells were filled as for Drosophila. For mouse, we ran ANOVA on pooled intensities from liver, kidney and hypothalamus. We also analyzed each tissue separately. The Tukey HSD procedure was performed in Matlab and generated a confidence interval for each pair of comparisons that has a family-wise error rate of, at most, $\alpha=0.05$ (see Additional data file 3).

For the Drosophila data, we applied a bootstrapped HodgesLehmann (HL) estimate of median differences to determine if $\mathrm{X}$-chromosome expression was more similar to autosomal expression or to expression from autosomal aberrations (see Additional data files 4,5). Additional data file 6 shows a cartoon depicting bootstrap HL estimates of median differences followed by the Kolmogorov-Smirnov (KS) test. HL estimates and KS tests were performed in Bioconductor. For ratiometric data (see Additional data file 4), the HL estimate was calculated for every drawing of 600 bootstrap samples from the $(D p /+) /(+/+)$ and AA/AA distributions to generate a new distribution of differences. Next, the same bootstrap sampling was used for the $\mathrm{XX} / \mathrm{X}$ and $\mathrm{AA} / \mathrm{AA}$ distribution to generate a second distribution of differences. Intensity data were treated similarly (see Additional data file 5). The two distributions of differences were then compared using the KS test. The differences in the medians and associated $D$ and $p$-values for the KS tests then permitted inference concerning the proximity of expression ratios or intensities. In practical terms, a significant $p$-value allows us to accept (not reject) the assumption that expression ratios $\mathrm{AA} / \mathrm{AA}$ are closer to $\mathrm{XX} / \mathrm{X}$ than to $\mathrm{Dp} /+$. This procedure of bootstrap sampling, HL estimation and KS evaluation was repeated ten times to check the stability and robustness of the inferences. In all cases, replicate evaluations resulted in the same inference.

\section{Additional data files}

The following files are available with the online version of this article. Additional data file 1 is a figure showing the effect of different data handling techniques on differential expression resulting from altered gene dose on the autosomes. Additional data file 2 is a figure showing the experimental design in detail. Additional data files 3-5 are tables showing the results of statistical analysis of gene expression ratios and absolute intensities for whole chromosome arms and for autosomal aneuploid segments: Additional data file 3 shows one-way ANOVA comparisons and Additional data file 4 shows estimation of Hodges-Lehmann (HL) median differences between expression ratios in various experiments and Additional data file 5 shows estimation of Hodges-Lehmann (HL) median differences between signal intensities. Additional data file 6 is a figure showing a simple picture depicting bootstrap HL estimates of median differences followed by the Kolmogorov-Smirnov (KS) test.

\section{Acknowledgements}

This research was supported in part by the Intramural Research Program of the NIH, NIDDK and CIT. We acknowledge our colleagues Mathias Beller, Jurrien Dean, Sandra Farkas, Leonie Hempel, Jamileh Jemison, Rasika Kalamegham, Alan Kimmel, James Minor, John E. Smith, Charles Vinson, and Yu Zhang for discussions and technical assistance. We are grateful to Christine Disteche for providing a preprint of work from her laboratory and to Jim Birchler for discussions on autosomal aneuploidy and dosage compensation.

\section{References}

I. Rosenbusch B: The incidence of aneuploidy in human oocytes assessed by conventional cytogenetic analysis. Hereditas 2004, 141:97-105.

2. Lindsley DL, Sandler L, Baker BS, Carpenter AT, Denell RE, Hall JC, Jacobs PA, Miklos GL, Davis BK, Gethmann RC, et al.: Segmental 
aneuploidy and the genetic gross structure of the Drosophila genome. Genetics 1972, 71:157-184.

3. Celniker SE, Wheeler DA, Kronmiller B, Carlson JW, Halpern A, Patel S, Adams M, Champe M, Dugan SP, Frise E, et al:: Finishing a whole-genome shotgun: release 3 of the Drosophila melanogaster euchromatic genome sequence. Genome Biol 2002, 3:research0079.1-0079.14.

4. Pauli D, Oliver B, Mahowald AP: The role of the ovarian tumor locus in Drosophila melanogaster germ line sex determination. Development 1993, I I 9:123-I34.

5. Lu J, Oliver B: Drosophila OVO regulates ovarian tumor transcription by binding unusually near the transcription start site. Development 200I, I 28: |67|-|686.

6. Regan $\mathrm{CL}$, Fuller MT: Interacting genes that affect microtubule function: the nc2 allele of the haywire locus fails to complement mutations in the testis-specific $\beta$-tubulin gene of Drosophila. Genes Dev 1988, 2:82-92.

7. Cline TW, Meyer B]: Vive la difference: males vs females in flies vs worms. Annu Rev Genet 1996, 30:637-702.

8. Baker BS, Gorman M, Marin I: Dosage compensation in Drosophila. Annu Rev Genet 1994, 28:49I-52I.

9. Meller V, Kuroda M: Sex and the single chromosome. Adv Genet 2002, 46: I-24.

I0. Rastelli L, Kuroda MI: An analysis of maleless and histone H4 acetylation in Drosophila melanogaster spermatogenesis. Mech Dev 1998, 71:107-II7.

II. Schüpbach T: Normal female germ cell differentiation requires the female $X$ chromosome to autosome ratio and expression of Sex-lethal in Drosophila melanogaster. Genetics 1985, 109:529-548.

12. Bachiller D, Sanchez L: Mutations affecting dosage compensation in Drosophila melanogaster: effects in the germline. Dev Biol 1986, I I 8:379-384.

13. Gartler SM, Liskay RM, Campbell BK, Sparkes R, Gant N: Evidence for two functional $X$ chromosomes in human oocytes. Cell Differ 1972, I:215-218.

14. Kelly WG, Schaner CE, Dernburg AF, Lee MH, Kim SK, Villeneuve AM, Reinke V: X-chromosome silencing in the germline of C. elegans. Development 2002, I 29:479-492.

15. Heard E, Clerc P, Avner P: X-chromosome inactivation in mammals. Annu Rev Genet 1997, 3 I:57 I-6I 0.

16. Meyer BJ: Sex in the worm: counting and compensating $\mathbf{X}$ chromosome dose. Trends Genet 2000, I 6:247-253.

I7. Ohno S: Sex Chromosomes and Sex-linked Genes. Berlin: SpringerVerlag; 1967.

18. Charlesworth $B$ : The evolution of chromosomal sex determination and dosage compensation. Curr Biol 1996, 6: I49-162.

19. Charlesworth B: The evolution of sex chromosomes. Science I99|, 25 I:1030-1033.

20. Charlesworth B: Model for evolution of $\mathbf{Y}$ chromosomes and dosage compensation. Proc Natl Acad Sci USA 1978, 75:56 I8-5622.

21. Adler DA, Rugarli El, Lingenfelter PA, Tsuchiya K, Poslinski D, Liggitt HD, Chapman VM, Elliott RW, Ballabio A, Disteche CM: Evidence of evolutionary up-regulation of the single active $X$ chromosome in mammals based on Clc4 expression levels in Mus spretus and Mus musculus. Proc Natl Acad Sci USA 1997, 94:9244-9248.

22. Oliver B: Genetic control of germline sexual dimorphism in Drosophila. Int Rev Cytol 2002, 2 I 9: I-60.

23. Steinmann-Zwicky M, Schmid H, Nothiger R: Cell-autonomous and inductive signals can determine the sex of the germ line of Drosophila by regulating the gene SxI. Cell 1989, 57:157-166.

24. Parisi M, Nuttall R, Edwards $P$, Minor J, Naiman D, Lu J, Doctolero M, Vainer M, Chan C, Malley J, et al.: A survey of ovary-, testis-, and soma-biased gene expression in Drosophila melanogaster adults. Genome Biol 2004, 5:R40.

25. Parisi M, Nuttall R, Naiman D, Bouffard G, Malley J, Andrews J, Eastman S, Oliver B: Paucity of genes on the Drosophila $\mathbf{X}$ chromosome showing male-biased expression. Science 2003, 299:697-700.

26. Ashburner M, Ball CA, Blake JA, Botstein D, Butler H, Cherry JM, Davis AP, Dolinski K, Dwight SS, Eppig JT, et al.: Gene Ontology: tool for the unification of biology. Nat Genet 2000, 25:25-29.
27. FlyBase: The FlyBase database of the Drosophila genome projects and community literature. Nucleic Acids Res 2003, 3 I:172-175.

28. Reinke V, Gil IS, Ward S, Kazmer K: Genome-wide germlineenriched and sex-biased expression profiles in Caenorhabditis elegans. Development 2004, I 3 I:3 I I-323.

29. Rinn JL, Rozowsky JS, Laurenzi IJ, Petersen PH, Zou K, Zhong W, Gerstein M, Snyder M: Major molecular differences between mammalian sexes are involved in drug metabolism and renal function. Dev Cell 2004, 6:79I-800.

30. Nguyen DK, Disteche CM: Dosage compensation of the active X chromosome in mammals. Nat Genet 2006, 38:47-53.

31. Chiang PW, Kurnit DM: Study of dosage compensation in Drosophila. Genetics 2003, I65: I 167-I I8I.

32. Jin W, Riley RM, Wolfinger RD, White KP, Passador-Gurgel G, Gibson G: The contributions of sex, genotype and age to transcriptional variance in Drosophila melanogaster. Nat Genet 200I, 29:389-395.

33. Arbeitman MN, Furlong EE, Imam F, Johnson E, Null BH, Baker BS, Krasnow MA, Scott MP, Davis RW, White KP: Gene expression during the life cycle of Drosophila melanogaster. Science 2002, 297:2270-2275.

34. Andrews J, Bouffard GG, Cheadle C, Lu J, Becker KG, Oliver B: Gene discovery using computational and microarray analysis of transcription in the Drosophila melanogaster testis. Genome Res 2000, 10:2030-2043.

35. Ranz JM, Castillo-Davis Cl, Meiklejohn CD, Hartl DL: Sexdependent gene expression and evolution of the Drosophila transcriptome. Science 2003, 300: I742-I745.

36. Birchler JA, Pal-Bhadra M, Bhadra U: Dosage dependent gene regulation and the compensation of the $X$ chromosome in Drosophila males. Genetica 2003, I I 7:179-190.

37. Pal Bhadra M, Bhadra U, Kundu J, Birchler JA: Gene expression analysis of the function of the male-specific lethal complex in Drosophila. Genetics 2005, I69:206|-2074.

38. Kai T, Williams D, Spradling AC: The expression profile of purified Drosophila germline stem cells. Dev Biol 2005, 283:486-502.

39. Hiller $M$, Chen $X$, Pringle $M J$, Suchorolski $M$, Sancak $Y$, Viswanathan S, Bolival B, Lin TY, Marino S, Fuller MT: Testis-specific TAF homologs collaborate to control a tissue-specific transcription program. Development 2004, I 3 I:5297-5308.

40. Bielinska B, Lu J, Sturgill D, Oliver B: Core promoter sequences contribute to ovo-B regulation in the Drosophila melanogaster germline. Genetics 2005, I69:16I-I72

4I. Ruiz MF, Esteban MR, Donoro C, Goday C, Sanchez L: Evolution of dosage compensation in Diptera: the gene maleless implements dosage compensation in Drosophila (Brachycera suborder) but its homolog in Sciara (Nematocera suborder) appears to play no role in dosage compensation. Genetics 2000, I56:1853-1865.

42. da Cunha PR, Granadino B, Perondini AL, Sanchez L: Dosage compensation in sciarids is achieved by hypertranscription of the single $\mathbf{X}$ chromosome in males. Genetics 1994, I 38:787-790.

43. Serna E, Gorab E, Ruiz MF, Goday C, Eirin-Lopez JM, Sanchez L: The gene Sex-lethal of the Sciaridae family (order Diptera, suborder Nematocera) and its phylogeny in dipteran insects. Genetics 2004, 168:907-921.

44. Hilfiker A, Hilfiker-Kleiner D, Pannuti A, Lucchesi JC: mof, a putative acetyl transferase gene related to the Tip60 and MOZ human genes and to the SAS genes of yeast, is required for dosage compensation in Drosophila. EMBO J 1997, 16:2054-2060.

45. Kelley RL, Wang J, Bell L, Kuroda MI: Sex lethal controls dosage compensation in Drosophila by a non-splicing mechanism. Nature 1997, 387:195-199.

46. Cline TW: Autoregulation functioning of a Drosophila gene product that establishes and maintains the sexually determined state. Genetics 1984, I07:23 I-277.

47. Smith ER, Allis CD, Lucchesi JC: Linking global histone acetylation to the transcription enhancement of X-chromosomal genes in Drosophila males. J Biol Chem 200I, 276:3 I 483-3 I 486. 
48. Oliver B, Singer J, Laget V, Pennetta G, Pauli D: Function of Drosophila ovo+ in germ-line sex determination depends on X-chromosome number. Development 1994, I 20:3 185-3195.

49. Sahut-Barnola I, Pauli D: The Drosophila gene stand still encodes a germline chromatin-associated protein that controls the transcription of the ovarian tumor gene. Development 1999, 126:1917-1926.

50. Andrews J, Garcia-Estefania D, Delon I, Lu J, Mevel-Ninio M, Spierer A, Payre F, Pauli D, Oliver B: OVo transcription factors function antagonistically in the Drosophila female germline. Development 2000, 127:88I-892.

51. Wakimoto BT, Lindsley DL, Herrera C: Toward a comprehensive genetic analysis of male fertility in Drosophila melanogaster. Genetics 2004, 167:207-216.

52. Birchler JA: A study of enzyme activities in a dosage series of the long arm of chromosome one in maize. Genetics 1979, 92:I2II-I229.

53. Birchler JA, Riddle NC, Auger DL, Veitia RA: Dosage balance in gene regulation: biological implications. Trends Genet 2005, 21:219-226.

54. Devlin RH, Holm DG, Grigliatti TA: Autosomal dosage compensation Drosophila melanogaster strains trisomic for the left arm of chromosome 2. Proc Natl Acad Sci USA 1982, 79: $1200-1204$.

55. Kahlem P, Sultan M, Herwig R, Steinfath M, Balzereit D, Eppens B, Saran NG, Pletcher MT, South ST, Stetten G, et al.: Transcript level alterations reflect gene dosage effects across multiple tissues in a mouse model of Down syndrome. Genome Res 2004, I 4: I258-I 267.

56. Bond U, Neal C, Donnelly D, James TC: Aneuploidy and copy number breakpoints in the genome of lager yeasts mapped by microarray hybridisation. Curr Genet 2004, 45:360-370.

57. Phillips JL, Hayward SW, Wang Y, Vasselli J, Pavlovich C, PadillaNash H, Pezullo JR, Ghadimi BM, Grossfeld GD, Rivera A, et al.: The consequences of chromosomal aneuploidy on gene expression profiles in a cell line model for prostate carcinogenesis. Cancer Res 200 I, 6I:8I43-8I49.

58. Ghosh S, Lucchesi JC, Manning JE: The non-dosage compensated $L S P I-\alpha$ gene of Drosophila melanogaster lies immediately downstream of the dosage compensated $L / 2$ gene. Mol Gen Genet 1992, 233:49-52.

59. Marin I, Siegal ML, Baker BS: The evolution of dosage-compensation mechanisms. BioEssays 2000, 22: | |06-| | |4.

60. de Wit E, Greil F, van Steensel B: Genome-wide HPI binding in Drosophila: developmental plasticity and genomic targeting signals. Genome Res 2005, I 5: I265- 1273.

61. Johnston R, Wang B, Nuttall R, Doctolero M, Edwards P, Lü J, Vainer M, Yue H, Wang X, Minor J, et al.: FlyGEM, a full transcriptome array platform for the Drosophila community. Genome Biol 2004, 5:RI9.

62. Churchill GA: Fundamentals of experimental design for cDNA microarrays. Nat Genet 2002, 32 Suppl:490-495.

63. Gentleman RC, Carey VJ, Bates DM, Bolstad B, Dettling M, Dudoit $\mathrm{S}$, Ellis B, Gautier L, Ge Y, Gentry J, et al.: Bioconductor: open software development for computational biology and bioinformatics. Genome Biol 2004, 5:R80.

64. Yang YH, Dudoit S, Luu P, Lin DM, Peng V, Ngai J, Speed TP: Normalization for CDNA microarray data: a robust composite method addressing single and multiple slide systematic variation. Nucleic Acids Res 2002, 30:el5.

65. Edgar R, Domrachev M, Lash AE: Gene Expression Omnibus: NCBI gene expression and hybridization array data repository. Nucleic Acids Res 2002, 30:207-210.

66. de Hoon MJ, Imoto S, Nolan J, Miyano S: Open source clustering software. Bioinformatics 2004, 20:1453-1454.

67. Deng W, Lin H: Asymmetric germ cell division and oocyte determination during Drosophila oogenesis. Int Rev Cytol 200I, 203:93-I38.

68. Mahowald AP: Assembly of the Drosophila germ plasm. Int Rev Cytol 200I, 203:187-2।3.

69. Nagoshi RN, Patton JS, Bae E, Geyer PK: The somatic sex determines the requirement for ovarian tumor gene activity in the proliferation of the Drosophila germline. Development 1995, | 2 1:579-587. 